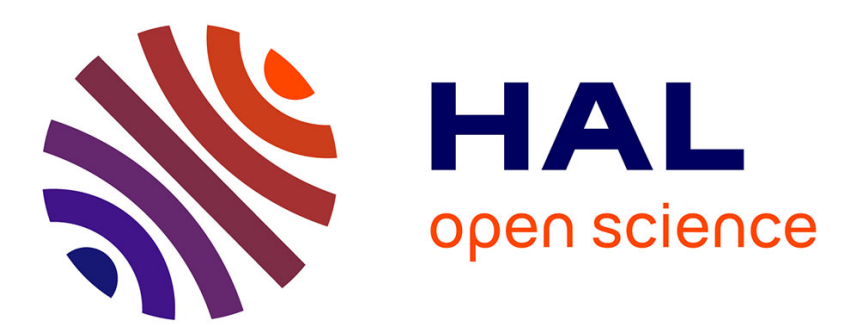

\title{
Bayesian parameter estimation of convective heat transfer coefficients of a roof-mounted radiant barrier system
}

Philippe Lauret, Frédéric Miranville, Harry Boyer, François Garde, Laetitia Adelard

\section{To cite this version:}

Philippe Lauret, Frédéric Miranville, Harry Boyer, François Garde, Laetitia Adelard. Bayesian parameter estimation of convective heat transfer coefficients of a roof-mounted radiant barrier system. Journal of Solar Energy Engineering, Transactions of the ASME, 2006, 128 (2), pp.213-225. hal00766246

\section{HAL Id: hal-00766246 \\ https://hal.science/hal-00766246}

Submitted on 17 Dec 2012

HAL is a multi-disciplinary open access archive for the deposit and dissemination of scientific research documents, whether they are published or not. The documents may come from teaching and research institutions in France or abroad, or from public or private research centers.
L'archive ouverte pluridisciplinaire HAL, est destinée au dépôt et à la diffusion de documents scientifiques de niveau recherche, publiés ou non, émanant des établissements d'enseignement et de recherche français ou étrangers, des laboratoires publics ou privés. 


\title{
Bayesian Parameter Estimation of Convective Heat Transfer Coefficients of a Roof-Mounted Radiant Barrier System
}

\author{
Philippe LAURET \\ e-mail : lauret@univ-reunion.fr \\ Frédéric MIRANVILLE \\ e-mail : frederic.miranville@univ-reunion.fr \\ Harry BOYER \\ e-mail : harry.boyer@univ-reunion.fr \\ François GARDE \\ e-mail : garde@univ-reunion.fr \\ Laetitia ADELARD \\ e-mail: adelard@univ-reunion.fr \\ University of Reunion Island, \\ Faculté des Sciences et Technologies, \\ Laboratoire de Génie Industriel, \\ Equipe Génie Civil et Thermique de l'habitat \\ BP 7151, 15 avenue René Cassin, \\ 97715 Saint-Denis, France
}

\begin{abstract}
This paper deals with the application of Bayesian methods to the estimation of two convective heat transfer coefficients of a roof-mounted radiant barrier system (RBS). As part of an empirical validation of the thermal model of the roofing complex, a parametric sensitivity analysis highlighted the importance of convective coefficients in the thermal behavior of a roofing complex. A parameter estimation method is then used in order to find the values of the coefficients that lead to an improvement of the thermal model. However, instead of using a classical parameter estimation method, we used a Bayesian inference approach to parameter estimation. The aim of the paper is to introduce the basic concepts of this powerful method in this simple two-parameter case. We show that Bayesian methods introduce an explicit treatment of uncertainty in modelling and a corresponding measure of reliability for the conclusions reached.

Keywords: Bayesian inference, radiant barriers, convective heat transfer coefficient, uncertainty, model validation
\end{abstract}

\section{Introduction}

In hot climates, action must be taken against solar heating to improve thermal comfort in buildings as well as to minimise the need for active cooling, which is responsible for heavy electricity consumption. Solar energy affects the whole building, but in particular the roof, because this surface is the most exposed to the sun. Therefore, if we wish to reduce the energetic contribution from the roof, several solutions are possible, one of which is thermal insulation. Mass insulation such as mineral wool and expanded foam (polyurethane and polystyrene) are generally used. These exhibit very low thermal conductivity, of the order of $0.05 \mathrm{~W} \cdot \mathrm{m}^{-1} \cdot \mathrm{K}^{-1}$ and therefore reduce heat transfer due to the conduction process.

However, this type of thermal insulation does not reduce radiative heat transfer particularly in the case of roofs which feature air layers. Devices which help to reduce heat transfer due to radiation are known under the generic name 'Radiant Barrier Systems', or RBS [1-4].

In order to assess the energetic performance of a building with a roofing complex that includes an RBS, we have designed a dedicated experimental set-up as well as a detailed thermal model representing this specific building. As part of the empirical validation of the thermal model, a parametric sensitivity analysis of the thermal model showed the importance of convective heat transfers through the roofing complex.

As a consequence, an optimization process has been carried out in order to find values of the convective heat transfer coefficients that lead to significant reduction in the residuals. As stated by Palomo del Barrio [5], the observation of large differences between nominal and estimated values indicates the presence of phenomena that are not properly taken into 
account by the model. Notice that these two steps (sensitivity analysis and optimization) are part of the methodology proposed in the framework of task 22 of the International Energy Agency (IEA) [6]. In this framework, three optimization methods have been proposed: the classical Gauss-Newton method, a Monte-Carlo approach and an heuristic bounding method. The latter takes into account data uncertainty.

In this paper, we propose the use of a Bayesian approach to parameter estimation [7]. The principles of Bayesian reasoning are outlined and applied to the estimation of two convective heat transfer coefficients of a roof-mounted radiant barrier system. In the Bayesian framework, uncertainties in parameter values and model output are naturally assessed. Error bars for the model output take into account in a natural way two contributions: one arising from the intrinsic noise in the data and one arising from uncertainties in the parameter values.

\section{RBS in a roofing complex}

The RBS are thin membranes, the faces of which are covered by a material with very low emissivity, which gives them a polished and therefore reflective appearance. Such surfaces release very little thermal radiation compared to the surfaces typically used in buildings. They also reflect much of the incident longwave radiation, and in this way significantly reduce the radiation heat transfer across the wall into which they have been inserted.

Figure 1 shows a standard roof including an RBS (hence giving the so-called roofing complex), installed according to current practice. This configuration represents a realistic situation, incorporating in particular details like the roofing framework. Further, the insertion of an RBS requires two air layers (upper and lower air layers), which in practise are made by fitting spacers, often made from parts of the roof framework itself. The roofing complex is composed of a corrugated covering made of galvanised steel (both sides are varnished dark blue), an RBS and a ceiling made of plasterboard. The framework includes rafters with a C-shaped profile and galvanised steel spacers on the lower face of the RBS. The lateral faces are made of dark-coloured galvanised steel sheets, except for the lower part of the roofing, for which the exterior separating face is made of an unpainted C-shaped panel. The roof is inclined at $20^{\circ}$ to the horizontal, which is the angle most frequently encountered.

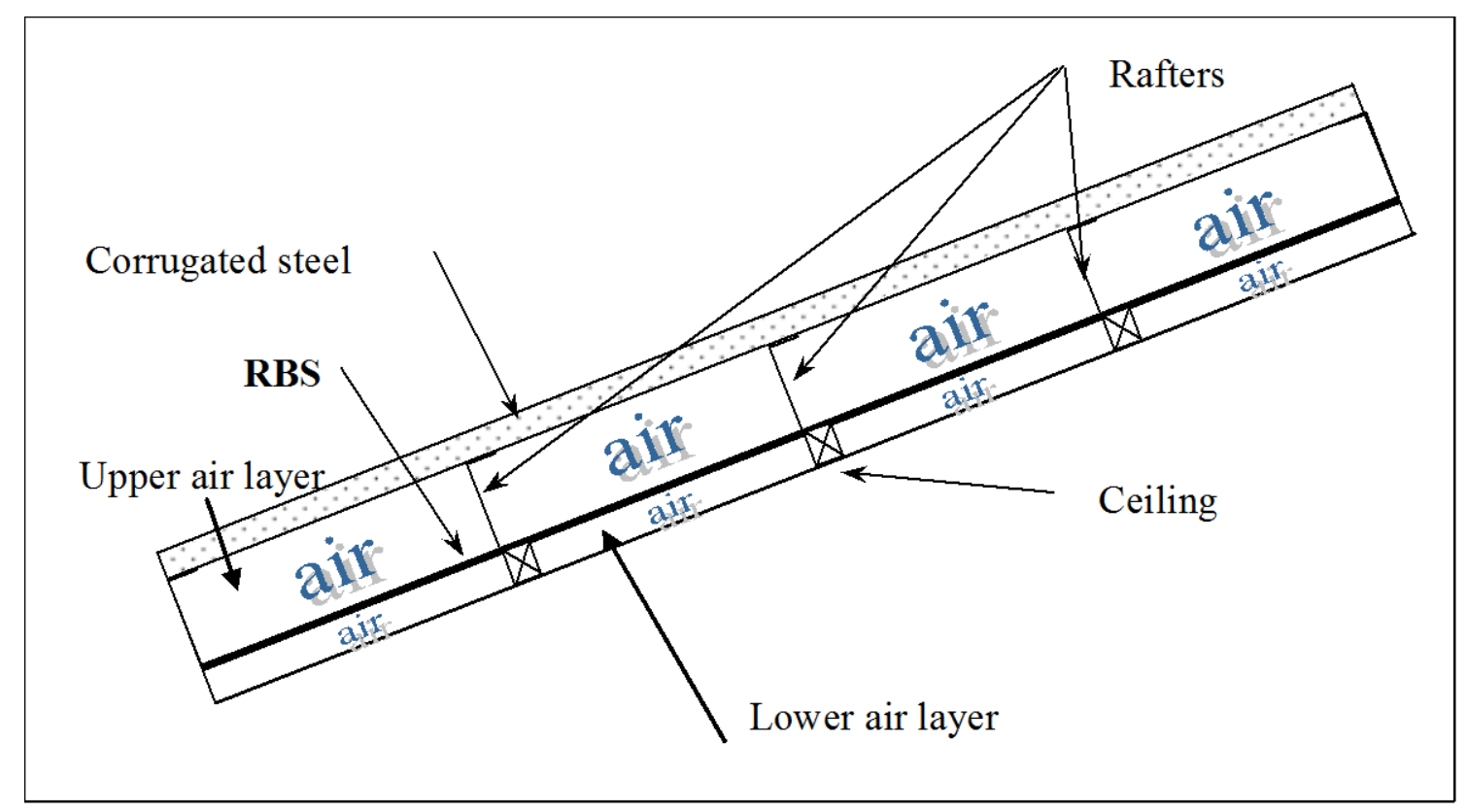

Fig. 1 Section view of the roofing complex

\section{Assessing the thermal behavior of the roofing complex}

In order to improve our understanding of the thermal behavior of the roofing complex, we designed a dedicated computerized model. An empirical validation exercise was then carried out in order to check model validity and to perform a model diagnosis [8]. This latter step allows parameters to be pinpointed i.e. the ones that are responsible for significant disagreements between model predictions and measurements. For the empirical validation exercise, a specific experimental set-up centered on a test cell that comprises a roofing complex was built.

\subsection{Description of the building}

The test cell has an interior volume of about $29.8 \mathrm{~m}^{3}$ and therefore resembles a typical room such as a bedroom or an office. The test cell is equipped with the roofing complex described in the previous section. The corrugated galvanised steel is a dark colour, which causes large solar heat transfer from the roof. Figure 2 shows a picture of the test cell. 


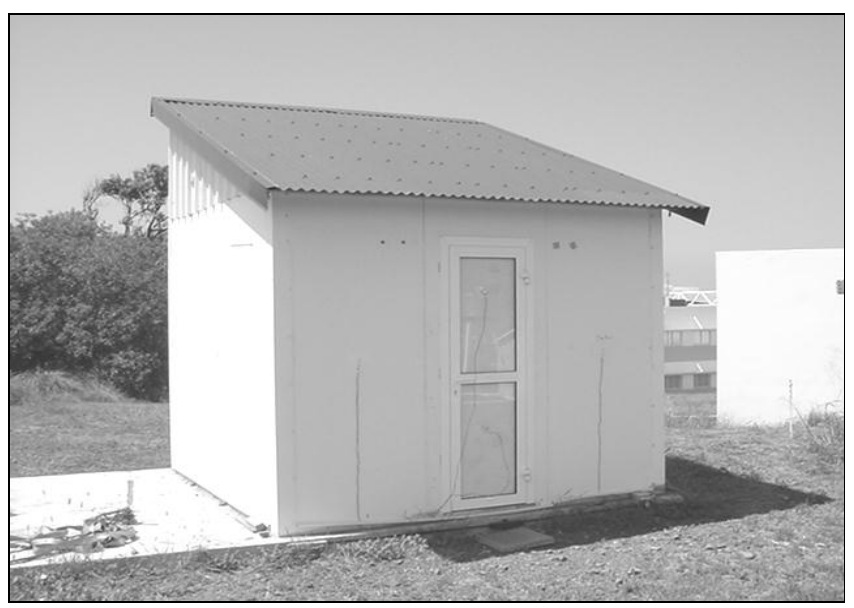

\subsection{Instrumentation}

Fig. 2 The test cell

In addition to the traditional measurements carried out in the test cell (surface temperature, indoor dry-bulb temperature, humidity), specific attention has been paid to the instrumentation of the roofing complex. This detailed instrumentation includes temperature measurements on the surface of the roof covering, on the RBS and on the ceiling, as well as on the rafters making up the roof framework. The dry-air and black globe temperatures of the air layers were also measured, and flux-meters allowed us to find the thermal flux across the roof, the RBS and the ceiling. The location and type of sensors used are shown in Fig. 3.

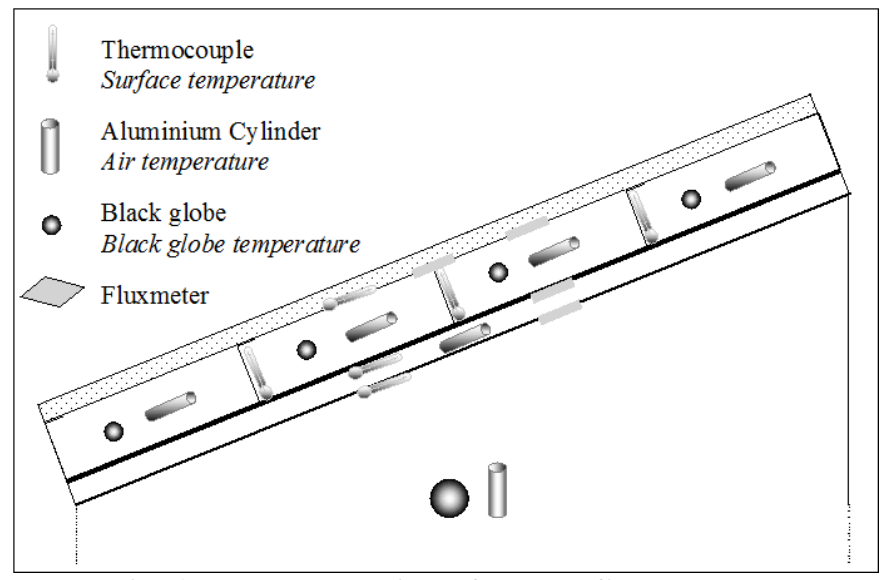

Fig. 3 Instrumentation of the roofing complex

\subsection{Model description}

Our study relies on CODYRUN [9], a detailed multizone building airflow and thermal simulation software regrouping design and research aspects. The thermal model of CODYRUN is based on the nodal analysis [10]. In other words, a lumped approach is used to represent the building thermal behavior. It is based on the analogy between the Fourier conduction equation and Ohm's law. The electrical network created by CODYRUN leads to a system of equations called state equations which, in the matrix formalism, has the following form (state space form):

$$
\begin{aligned}
& C(\theta) \frac{d T(t)}{d t}=A(\theta) T(t)+B(\theta) U(t) \\
& Y(t)=H T(t)
\end{aligned}
$$

where $T(t)(n x l)$, the state vector, comprises the temperatures at the $n$ nodes of the discretization mesh and $U(t)(r x l)$ is the vector of solicitations or $r$ input variables. $C(n x n)$ is a diagonal matrix of thermal capacities at the discretization nodes, $A(n x n)$ is a square symmetric matrix of parameters describing the thermal exchanges between nodes and $B$ (nxr) is a matrix of coupling parameters between the building and its environment. The output matrix $H$ (formed by zeros and ones) serves to select model outputs $Y(t)$.

Typical inputs for thermal models are the outdoor temperature, the solar irradiance on the different faces of the building and the heating or cooling power injected in the different zones of the building [6]. 
The vector of model parameters $\theta$ typically includes geometrical parameters (volume, surfaces, wall thickness, ...), thermophysical parameters (thermal conductivity, heat capacity ...), optical parameters (absorptance, reflectance, emissivities, ...), convective and radiative parameters, etc.

The output of the thermal model is usually the indoor dry air temperature $T_{a i}$ of the test cell and the associated residual (measurement minus prediction) serves as a criterion to validate the model. Nonetheless, as the survey concerns the thermal behavior of a roofing complex, one must not rely solely on this criterion. Therefore, in addition to the classical output $T_{a i}$, the output vector $Y(t)$ comprises also the indoor dry air temperature of the upper and lower air layers (see Fig. 1), namely $T_{u p}$ and $T_{l o}$. In the rest of the paper, we restrict ourselves to the study of $T_{l o}$ as our intention is to illustrate the application of Bayesian reasoning to the estimation of the convective coefficients of the lower air layer. For more detailed results and particularly those related to the upper air layer, the interested reader is referred to [8,11].

\subsection{Assumptions}

The given nodal analysis assumes that heat transfer by conduction through walls is mono-dimensional. Outdoor convective coefficients are based on the correlation of Cole and Sturrock [12]. Indoor and outdoor radiation heat transfer are linearized and the radiative exchange coefficients are identical for each wall. Indoor convective exchange coefficients are constant for each wall of the envelope. However, a specific correlation [13] given by Eq. (2) has been chosen to describe the convective heat transfer of the air layers of the roofing complex.

$$
h_{c i}=\Delta T^{0.425}+1.7
$$

where $\Delta T$ is the temperature difference at the surface-air interface.

\subsection{Appraisal of the model}

A 6 day-long experiment was carried out in February 2002. Figure 4 shows some of the meteorological conditions measured during the experiment. It must also be noticed that no special internal load was imposed inside the test cell. Predictions of the thermal model are shown in Fig. 5 and Fig. 6. It must emphasized that this model (with the convective heat transfer correlation given by Eq. (2)) was not calibrated to the data.
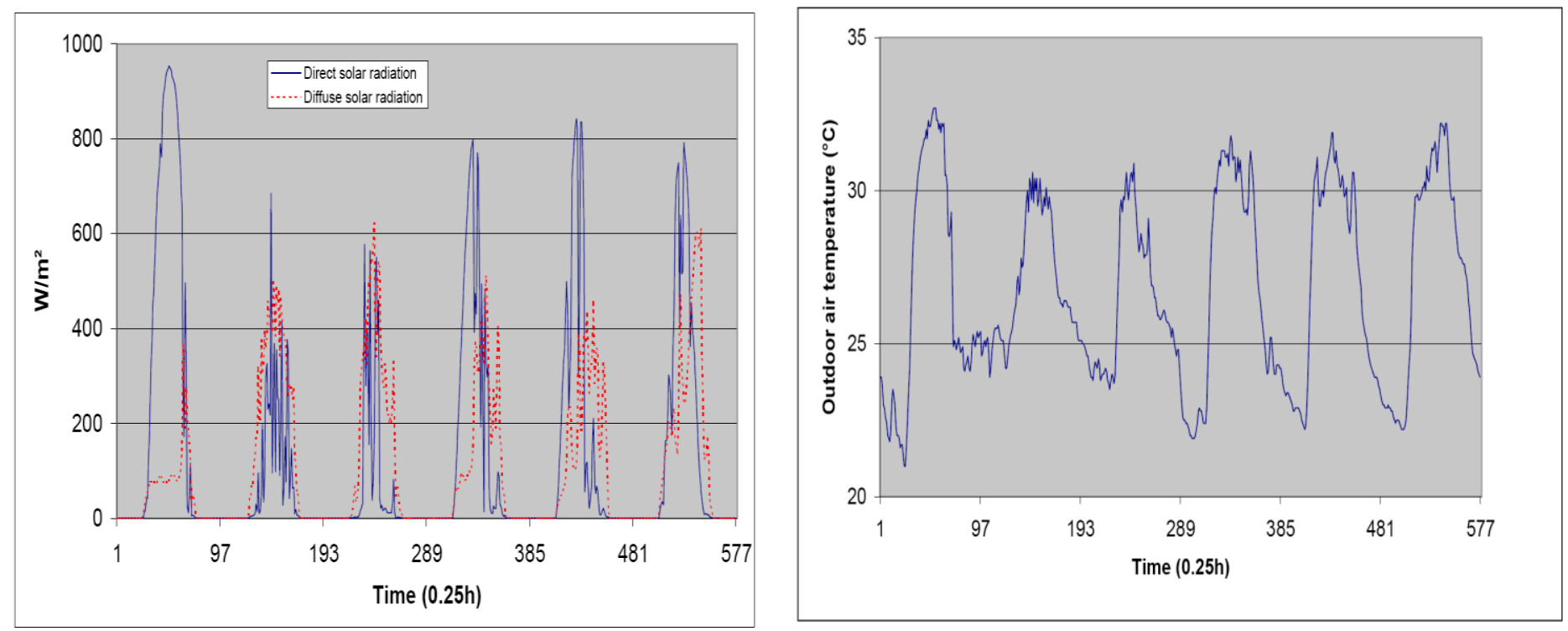

Fig. 4 Some meteorological variables measured during the experiment 

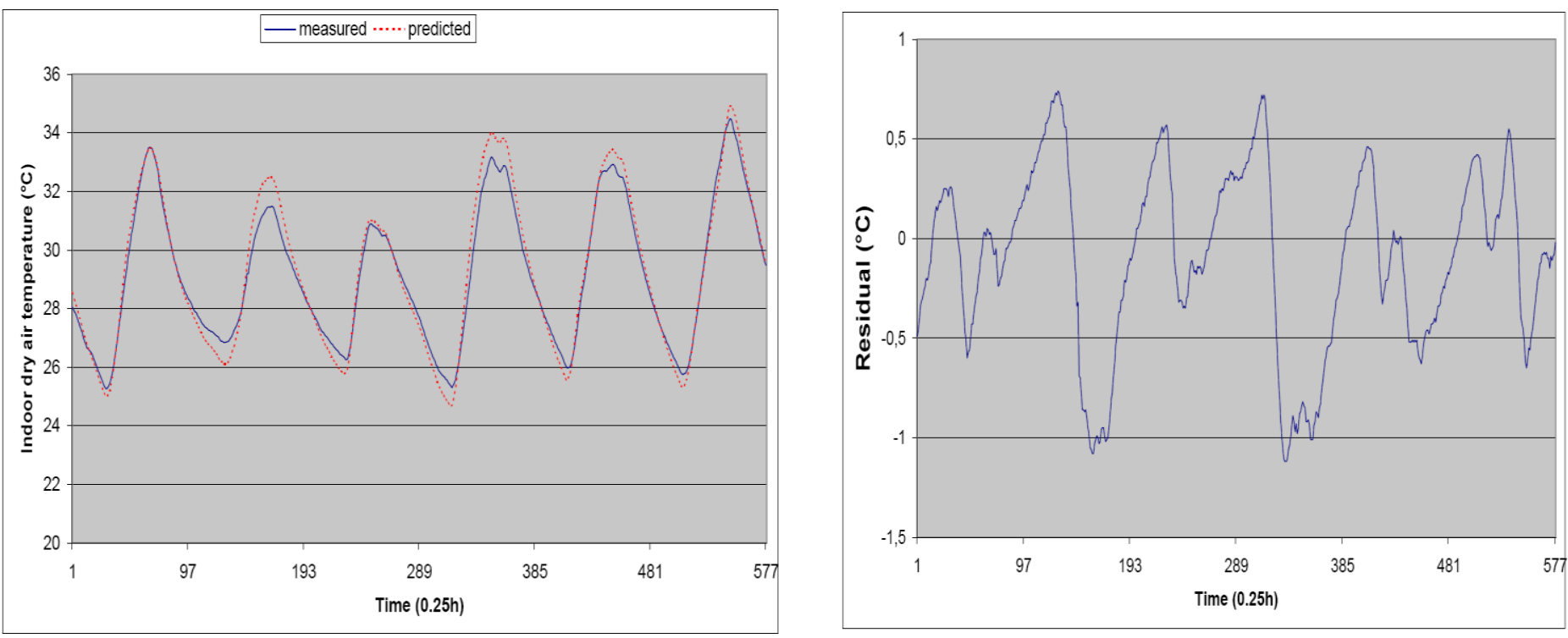

Fig. 5 Comparison between measurement and prediction of the indoor dry air temperature of the test cell $T_{a i}$
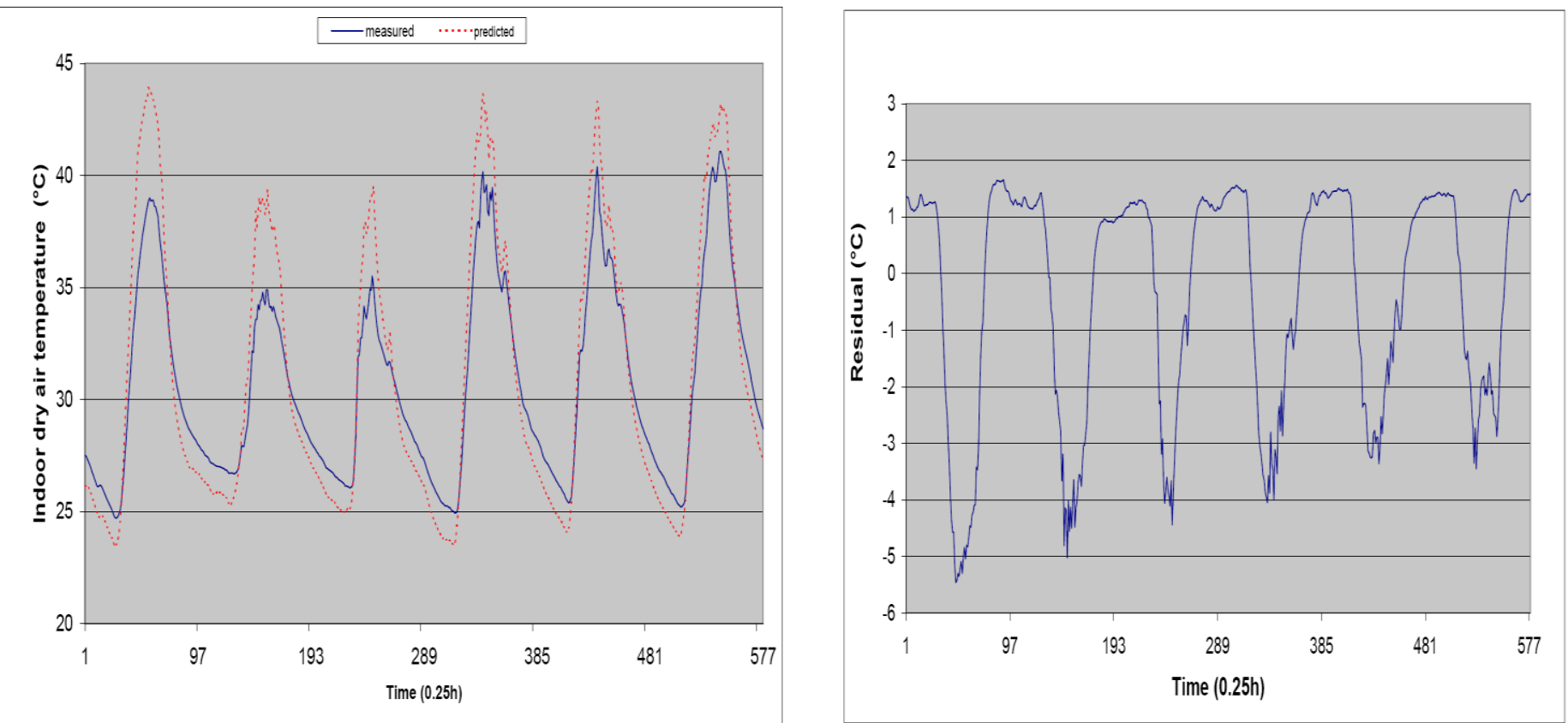

Fig. 6 Comparison between measurement and prediction of the indoor dry air temperature of the lower air layer $T_{l o}$

In the energy and building performance simulation field, in current practice, a thermal model is said to be in good agreement with experiment when the residual falls within the measurement uncertainty interval. The latter is characterized by upper and lower bounds (usually $\left[-0.5^{\circ} \mathrm{C}+0.5^{\circ} \mathrm{C}\right]$ ) that are derived from sensor manufacturer data. These bounds can however be increased in order to take into account a specific experimental set-up. These bounds express the extreme values of the residuals that are considered acceptable by the analyst [6]. A previous work [11] decided to consider the model valid if the residuals were contained in an interval bounded by $10 \%$ of the maximum amplitude of the output of interest (i.e here $T_{a i}$, $T_{l o}$ ). This choice leads to the following intervals given in Table 1. 
Table 1 Model validity criteria

\begin{tabular}{cc}
\hline $\begin{array}{c}\text { Model } \\
\text { output }\end{array}$ & Validity criterion \\
\hline$T_{a i}$ & $-1{ }^{\circ} \mathrm{C}<\operatorname{residual} T_{a i}<+1{ }^{\circ} \mathrm{C}$ \\
& \\
$T_{l o}$ & $-2{ }^{\circ} \mathrm{C}<\operatorname{residual} T_{l o}<+2{ }^{\circ} \mathrm{C}$ \\
\hline \hline
\end{tabular}

However, this type of validation criterion is quite misleading. The assumption that model predictions deviate from observations only because of error in the measurements implies an excessive faith in model validity. In reality, one also has to take into account model errors that result from imperfect system representation (model parameters and/or model structure). In other words, it is necessary to compute error bars on model output that take into account the measurement noise on the data as well as uncertainties in model parameters (it must be noticed that usually the model structure is deemed correct and the model inputs are noise free). Hence, a better criterion would consist in seeing if the measured values fall within the model output uncertainty bounds (also called the simulation or prediction uncertainty interval).

In fact, in the framework of Task 22 (Building Energy Analysis Tool) [5,6], several tools have been designed in order to check model validity and a model is said to be good enough when a significant overlapping is observed between model predictions and measurements uncertainty intervals. Among these tools, the use of Monte Carlo simulations enables the computation of the $x \%$ model output uncertainty bands. For instance, if measurements are outside the $99 \%$ simulation uncertainty bands (more than $1 \%$ of measured values falling outside these bands), one can conclude that the model is deficient. In the proposed methodology, in order to assess the model output uncertainties through the Monte Carlo simulations, one has to assign a priori uncertainty intervals to the parameters. For instance, the output uncertainty intervals may be given by the variation of $10 \%$ about the nominal value of the parameters.

In section 5, we will see that the Bayesian approach may offer a natural framework and sound theoretical basis for assessing uncertainties in model parameters and model output.

Nonetheless, according to the criterion given in Table 1, residuals for the indoor dry air temperature of the test cell (see Fig. 5) seem reasonable (the mean value of the residuals is $-0.09{ }^{\circ} \mathrm{C}$ and its standard deviation is $0.45{ }^{\circ} \mathrm{C}$ ) and tend to confirm that one must not rely only on this sole criterion in order to validate the roofing complex. Conversely, the residuals (see Fig. 6) for the lower air layer (the mean value of the residuals is $0.37{ }^{\circ} \mathrm{C}$ and its standard deviation is $2.01{ }^{\circ} \mathrm{C}$ ) show great discrepancies between measured and predicted values and one has to conclude that the model of the lower air layer of the roofing complex is incorrect.

Thus, the next objective, namely sensitivity analysis, is to identify the most important parameters in the model, which the modeller must know accurately to provide significant model behavior improvement.

\section{Parametric sensitivity analysis of the thermal model}

As mentioned above, Task 22 proposes a method for performing a sensitivity analysis of the thermal model. Nonetheless, our research team has developed a new sensitivity analysis method [14]. The method allows the identification of the most influential parameters of a given model. From one run to another, the parameters are changed according to a sinusoidal oscillation at distinct frequencies. Spectral analysis can then be used to assess the influence of the parameters on the model response (see Fig. 7). The remainder of this section summarizes the sensitivity analysis method as proposed by Mara et al. [14]. 


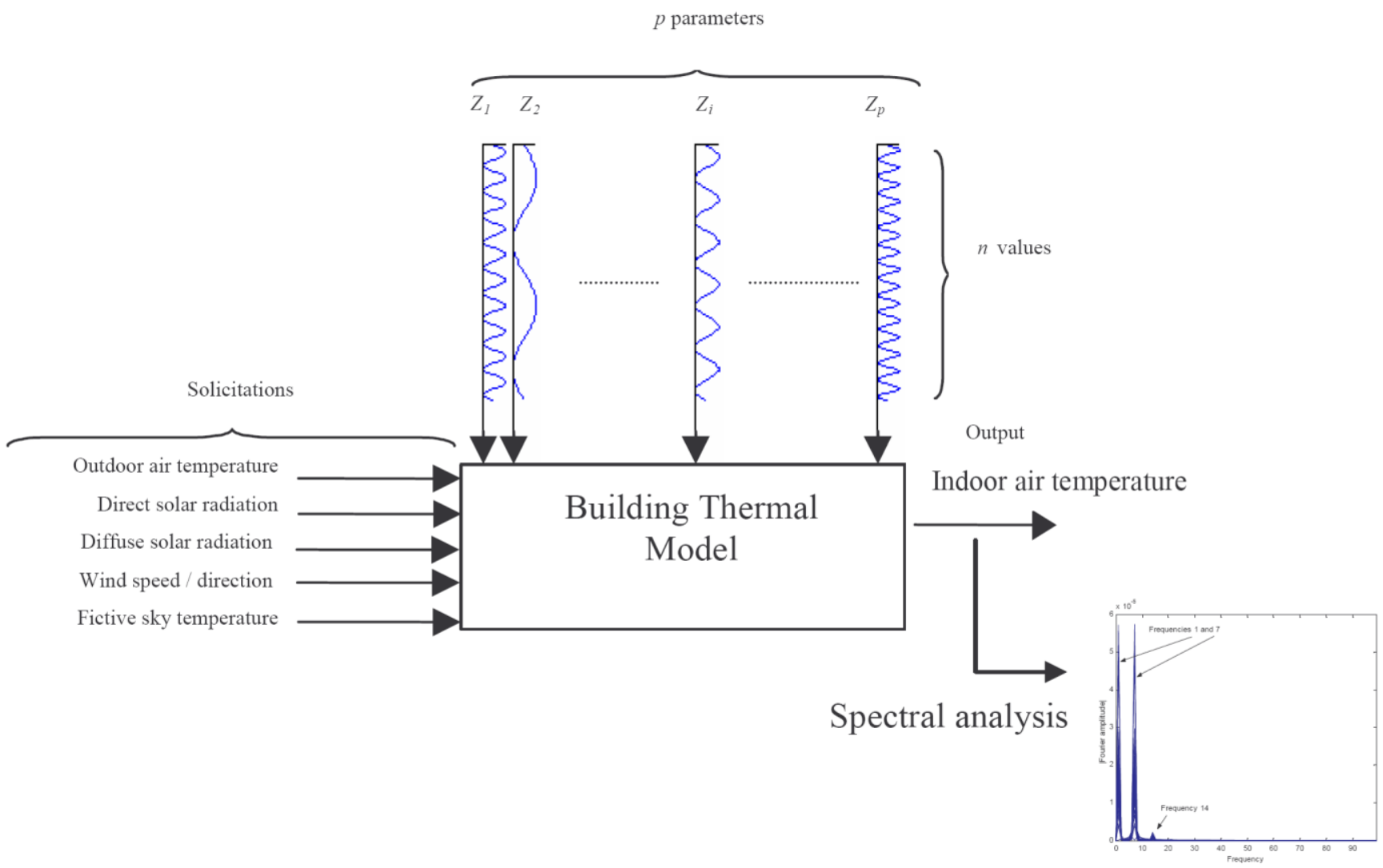

Fig. 7 Sketch of the parametric sensitivity analysis

\subsection{The method}

Consider a $p$-parameter model $y=F\left(Z_{1}, Z_{2}, \ldots, Z_{p}\right)$. To introduce the sensitivity analysis, it is convenient to consider, without loss of generality, that the model response under interest can be expressed in the form of the following polynomial expansion:

$$
y=y_{0}+\sum_{i=1}^{p} \beta_{i} z_{i}+\sum_{i=1}^{p} \sum_{j=1}^{p} \beta_{i j} z_{i} z_{j}+\ldots
$$

where $z_{i}$ is the standardized parameter (between $[-1 ;+1]$ ) relative to $Z_{i}$, the $\beta_{i}$ 's are the first order regression coefficients, the $\beta_{i j}$ 's are the second order regression coefficients, and the $z_{i} z_{j}$ represent the first-order interaction between the parameters $i$ and $j$.

Let us suppose that $n$ simulation runs of the model are performed by varying each factor as follows:

$$
Z_{i}^{k}=Z_{i}^{0}+\lambda_{i} \sin \left(2 \pi f_{i} \frac{k}{n}\right)
$$

where $u=k / n=\{0,1 / n, 2 / n, \cdots,(n-1) / n\}, Z_{i}^{0}$ is the nominal value, $\lambda_{i}$ is the oscillation amplitude, $f_{i}$ is the driving frequency and $n$ is the total number of simulation runs and $k$ is the simulation number.

In the proposed approach, the number of simulation runs $n$ equals the sampling frequency and therefore must be greater than $2 \times \max _{i \in[1, p]}\left(f_{i}\right)$ to satisfy the Nyquist Criterion.

It is readily shown that the standardization of $Z_{i}$ leads to $z_{i}^{k}=\sin \left(2 \pi f_{i} \frac{k}{n}\right)$ and that Eq. (3) becomes:

$$
y=y_{0}+\sum_{i=1}^{p} \beta_{i} \sin \left(2 \pi f_{i} u\right)+\sum_{i=1}^{p} \sum_{j=1}^{p} \beta_{i j} \sin \left(2 \pi f_{i} u\right) \sin \left(2 \pi f_{j} u\right)+\varepsilon
$$

By neglecting the error term $\varepsilon$ (i.e only first-order interactions between parameters are taken into account), we see that: 


$$
\Delta y \square y-y_{0}=\underbrace{\sum_{i=1}^{p} \beta_{i} \sin \left(2 \pi f_{i} u\right)}_{\text {term } 1}+\underbrace{\sum_{i=1}^{p} \sum_{j=1}^{p} \frac{\beta_{i j}}{2}\left(\cos \left(2 \pi\left(f_{i}-f_{j}\right) u\right)-\cos \left(2 \pi\left(f_{i}+f_{j}\right) u\right)\right)}_{\text {term } 2}
$$

$\Delta y$ represents the variations of the output around the base case response, due to the oscillation of the parameters according to Eq. (4). Term 1 contains the main effects, term 2 represents the first-order interactions, $\beta_{i}$ is the measure of the effect of the parameter $i$ only upon the output and $\beta_{i j}$ is the interaction effect between parameters $\mathrm{i}$ and $\mathrm{j}$ upon the output.

In fact, if the model is time-dependent, $\Delta y$ is an $m \times n$ matrix where $m$ is the number of simulation time steps. Thus, the evaluation of a parameter's influence must be performed at each time step.

Several qualitative inferences can be drawn by visualising the Fourier transform of Eq. (6):

1. The more a parameter is influential, the higher the amplitude of its assigned frequency

2. First-order interaction (between two parameters) induces two other frequencies which are the sum and the difference of the interacting parameters' driving frequencies.

3. The induced frequencies may interfere with the original frequencies. As a consequence, the set of frequencies assigned to the parameters must be properly chosen.

A quantitative analysis of the importance of the parameters can be made by estimating the regression coefficients. Because sine and cosine are orthogonal functions, the effects of the parameters can be easily estimated through the following relationships:

$$
\begin{gathered}
\beta_{i}=\frac{2}{n} \sum_{k=0}^{n-1} \Delta y \sin \left(2 \pi f_{i} \frac{k}{n}\right) \\
\beta_{i j}=\frac{4}{n} \sum_{k=0}^{n-1} \Delta y \cos \left(2 \pi\left(f_{i}-f_{j}\right) \frac{k}{n}\right)=-\frac{4}{n} \sum_{k=0}^{n-1} \Delta y \cos \left(2 \pi\left(f_{i}+f_{j}\right) \frac{k}{n}\right)
\end{gathered}
$$

\subsection{Application to the thermal model of the test cell}

All the thermo-physical, geometric, radiative and convective properties of the faces are taken into account by the analysis. Each parameter is assigned a different driving frequency and is varied sinusoidally by $\pm 10 \%$ around its base case value. Notice however that, in order to perform the sensitivity analysis, the convective heat transfer coefficients of the lower air layer must be represented by base case values (see Table 2). Hence, the latter have been inferred from Eq. (2). Indeed, these base case values correspond to the mean of the dynamic variation of these parameters represented by Eq. (2). As the highest frequency assigned is 687 , the number of simulation runs is given by $n=2 \max _{i \in[1, p]}\left(f_{i}\right)+1=1375$.

Table 2 Base case values of coefficients for interior convection heat exchanges used for the parametric sensitivity analysis

\begin{tabular}{cc}
\hline \hline Location & Base case value $: h_{\text {ci,base }}\left(W \cdot m^{-2} \cdot K^{-1}\right)$ \\
\hline Lower roofing faces & 3 \\
Upper lateral air layer faces & 3 \\
Upper RBS faces & 3 \\
Lower lateral air layer faces & 0.5 \\
Lower RBS faces & 1 \\
Top-side of ceiling & 1 \\
Lower-side of ceiling & 1 \\
Body walls & 3.5 \\
\hline \hline
\end{tabular}

As we focus on the thermal behavior of the roofing complex, results of the parametric sensitivity analysis are only given for the lower air layer. Again, for more detailed results, the interested reader is referred to [8,11].

For the air temperature of the lower air layer $T_{l o}$, the important parameters are given in Table 3 . The ranking of the parameters has been done firstly by qualitatively analyzing the Fourier transform of $\Delta T_{l o}$ (i.e. the lower air layer temperature 
variations as in our application, $y=T_{l o}$ ) and secondly by quantitatively evaluating the effects of the parameters i.e. the regression coefficients $\beta_{i}(t)$. Notice that for the present application, the interaction effects $\beta_{i j}(t)$ were negligible. Figure 8 (a), taken from the work of [11], shows the Fourier transform of Eq. (6) and Fig. 8 (b) displays the hourly regression coefficients (given by Eq. (7)) of the most important parameters.

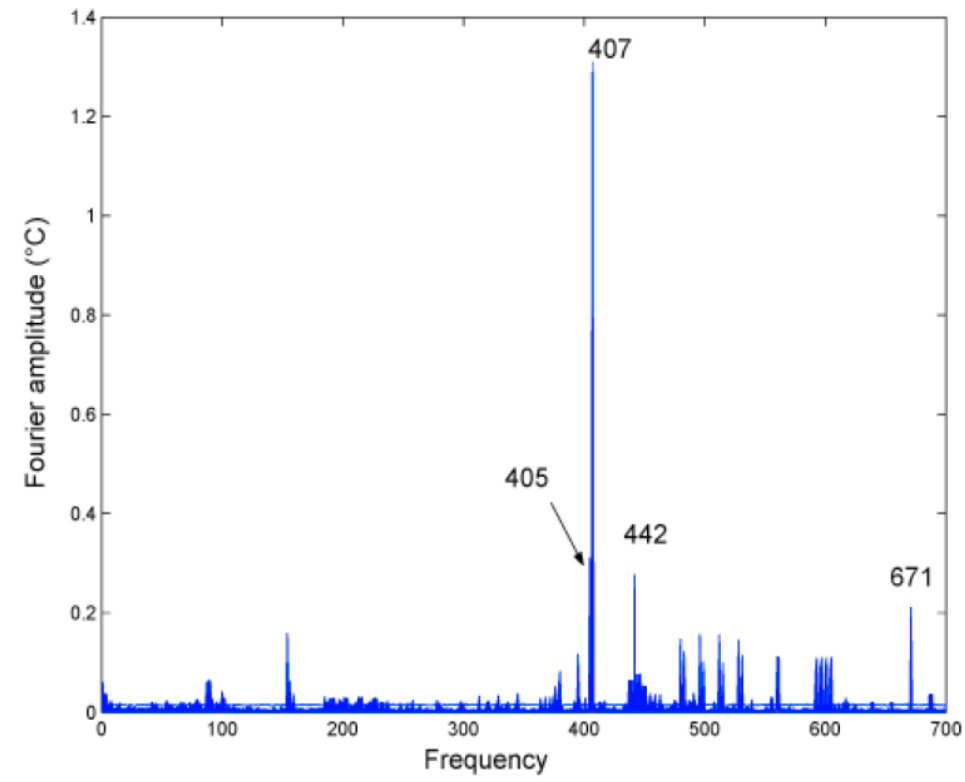

(a)

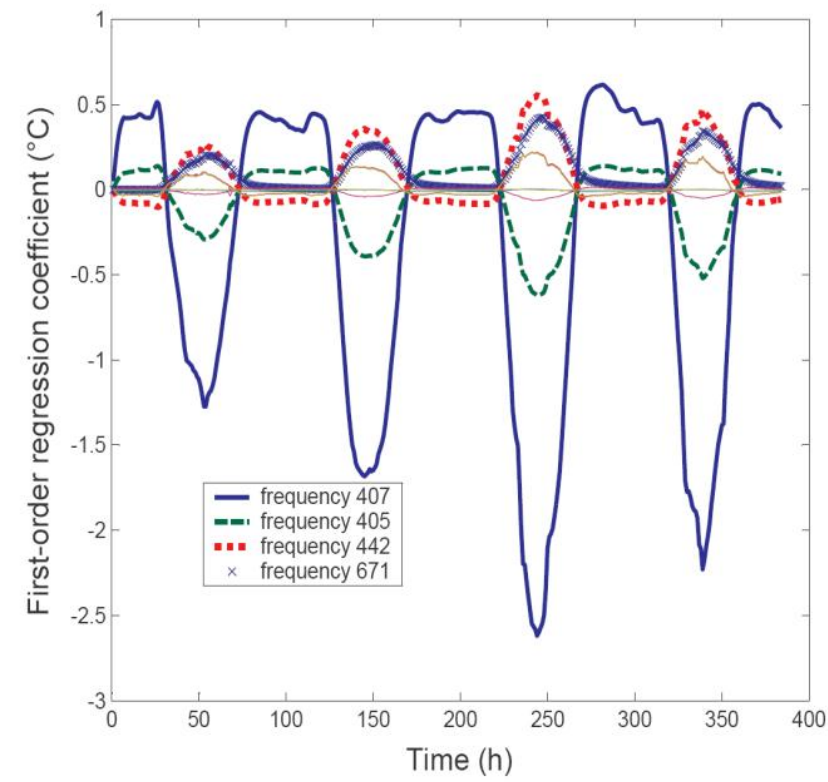

(b)

Fig. 8 Fourier Transforms of $\Delta T_{l o}$ (a) and effects of some important factors (b)

Table 3 The most influential parameters (with their assigned frequency) for the indoor dry air temperature of the lower air layer $T_{l o}$

\begin{tabular}{rll}
\hline $\begin{array}{r}\mathrm{r} \\
\text { ank }\end{array}$ & $\begin{array}{c}\text { freque } \\
\text { ncy }\end{array}$ & \multicolumn{1}{c}{ Parameter } \\
\hline 1 & 407 & Upper convective heat exchange coefficient of the ceiling \\
2 & 405 & Surface area of the ceiling \\
3 & 442 & Lower convective heat exchange coefficient of the RBS \\
4 & 671 & Absorptivity of the roof \\
\hline \hline
\end{tabular}

For this output, we can see that the most influential parameters are the upper convective heat exchange coefficient of the ceiling, and its surface area. Thus, it seems that the convective flux is highlighted by the method. The convective heat exchange coefficient of the RBS lower face also appears, but its influence is less important.

In the following, we focus on the determination of the convective heat transfer coefficients of the lower air layer. The next section is devoted to a Bayesian identification of these two parameters.

\section{Bayesian inference}

Bayesian probability theory is currently experiencing an increase in popularity in the sciences as a means of probabilistic inference [15]. Cox [16] showed that any method of scientific inference that satisfies simple rules of logical and consistent reasoning must be equivalent to the use of ordinary probability theory as originally developed by Bayes [17] and Laplace [18]. Two of these simple rules of probability theory are the sum rule and product rule (where prob stands either for a probability or a probability density function (pdf) ):

$$
\operatorname{prob}(x \mid I)+\operatorname{prob}(\bar{x} \mid I)=1
$$




$$
\operatorname{prob}(x, y \mid I)=\operatorname{prob}(x \mid y, I) \times \operatorname{prob}(y \mid I)
$$

where $\bar{x}$ represents the proposition that $x$ is false, the vertical bar "|" means "given" and the comma is read as the conjunction "and" [19]. Two useful relationships are derived from these basic rules, namely, Bayes's theorem and the marginalization relationship:

$$
\begin{gathered}
\operatorname{prob}(x \mid y, I)=\frac{\operatorname{prob}(y \mid x, I) \times \operatorname{prob}(x \mid I)}{\operatorname{prob}(y \mid I)} \\
\operatorname{prob}(x \mid I)=\int \operatorname{prob}(x, y \mid I) d y
\end{gathered}
$$

where the symbol I denotes the relevant background and assumptions. Notice that, for sake of clarity, the relevant background $I$ will be omitted in the subsequent formulae related to the pdf's.

In the Bayesian context, a probability represents a degree-of-belief (or encodes a state of knowledge); that is, how likely something is to be true based on all the relevant information at hand.

However, this concept seems too vague and too subjective to the school of conventional statistics (i.e. a frequentist approach) which defined probability as the long-run relative frequency with which an event occurred, given infinitely many repeated experimental trials. Indeed, the concept of degree-of-belief is criticized by the school of frequentists as it leads to subjectivity (because my belief could be different from yours). Although the frequency definition appears to be more objective, it fails to tackle most real-life scientific problems. Further, from a Bayesian viewpoint, all probabilities are always conditional (i.e. based on all the relevant background) and as stated by Jaynes [20], objectivity requires only that two people having the same information should assign the same probability. A good review of the Bayesian approach is given by [21,22].

Bayesian methods can be used either for model selection problems [23] or for parameter estimation problems [7]. As Bayesian probability theory does not define a probability as a frequency of occurrence but rather as a reasonable degree of belief, it is possible to assign probabilities to propositions such as "The probability that parameter $\theta$ had value $x$ when data was taken". In other words, in the Bayesian framework, questions of the form : "What is the best estimate of a parameter one can make from the data and prior information?" make perfect sense.

In a modeling application, Bayesian inference deals with the estimation of the values of $m$ model parameters $\theta=\left(\theta_{1}, \theta_{2}, \cdots, \theta_{m}\right)$ about which there may be some prior beliefs. These prior beliefs can be expressed as a probability density function (pdf) called prior, $p(\theta)$ and may be interpreted as the probability placed on all possible parameter values before collecting any new data. The dependence of the $N$ observations (or measurements) $D=\left(d_{1}, d_{2}, \cdots, d_{N}\right)$ on the $m$ parameters $\theta$ can also be expressed as a pdf : $p(D \mid \theta)$, called the likelihood function. The latter is used to update the prior beliefs about $\theta$, to account for the new data $D$. This updating is done through Bayes's theorem :

$$
p(\theta \mid D)=\frac{p(\theta) p(D \mid \theta)}{\int_{\theta} p(\theta) p(D \mid \theta) d \theta}
$$

where $p(\theta \mid D)$ represents the posterior pdf and expresses the values of the parameter after observing the new data. In other words, the prior is modified by the likelihood function to yield the posterior.

As the denominator in Eq. (13) does not depend on $\theta$ and therefore acts as a normalization constant, Bayes's theorem is often expressed as :

$$
p(\theta \mid D) \propto p(\theta) p(D \mid \theta)
$$

A major difference between Bayesian methods and classical parameter estimation methods (least squares or maximum likelihood) is that Bayesian inference yields the entire posterior pdf and not just a single best value that optimizes an objective function. The former obviously convey much more information than the latter (see discussion below in subsection marginal distributions).

In the Bayesian framework, uncertainties in parameter values and model output are naturally assessed. For instance, the position of the maximum of the posterior pdf represents a best estimate of the parameter; its width or spread about this optimal value gives an indication of the uncertainty in the estimate of the parameters. Error bars for the model output take into account in a natural way two contributions: one arising from the intrinsic noise in the data and one arising from uncertainties in the parameter values given by the width of the posterior pdf.

In this paper, the principles of Bayesian reasoning are outlined and applied to the estimation of the two convective heat transfer coefficients of the lower air layer of the roofing complex. 


\subsection{A Bayesian approach to estimating the convective heat transfer coefficients}

\section{The likelihood function}

Let us consider the following form:

$$
d=y+\varepsilon
$$

where $d$ represents the experimental measurements of the indoor dry air temperature of the lower air layer $T_{l o}$, $y=f(\theta, t)$ the ideal (noiseless) thermal model response that corresponds to the output of interest, namely $T_{l o}$ and where the noise $\varepsilon$ is an expression of the various uncertainties (i.e. measurement noise plus modeling error). The vector $\theta=\left[h_{1} h_{2}\right]$ represents the two convective heat transfer coefficients of the lower air layer.

If the noise in the data is assumed to be Gaussian with variance $\sigma^{2}$, independent and additive [7] then the likelihood function takes the following form:

$$
p(D \mid \theta)=\frac{1}{\left(2 \pi \sigma^{2}\right)^{N / 2}} \exp \left(-\frac{1}{2 \sigma^{2}} S(\theta)\right)
$$

where $N$ represents the number of experimental measurements and the term $S(\theta)=\sum_{k=1}^{N}\left(d_{k}-y_{k}\right)^{2}$ is the sum of the squares of the residuals.

Notice that the mathematical model (and the associated underlying assumptions) used to represent the functional relationship $f$ between the parameters $\theta$ and the noiseless data $y_{k}$ is part of the relevant background information $I$.

It is also important to note that in the preceding analysis the size (or value) of $\sigma$ is not (a priori) known. Furthermore, according to Qian [24], specifying an appropriate value for $\sigma$ in advance is difficult and can strongly influence the estimation of model parameters. One way of dealing with this problem is to include the noise in the list of parameters.

\section{The prior}

The prior pdf represents what is known about the parameters (convective heat transfer coefficients and the value of the noise) before collecting the data. Therefore, this prior information is represented by the following pdf (assuming independence between parameters):

$$
p(\theta, \sigma)=p(\theta) \times p(\sigma)=p\left(h_{1}\right) \times p\left(h_{2}\right) \times p(\sigma)
$$

One clear prior information is that the coefficients are positive; this information can be encoded through the simple expression (i.e. flat prior)

$$
p\left(h_{1}\right) \text { or } p\left(h_{2}\right)=\left\{\begin{array}{c}
\text { constant for } h_{1} \text { or } h_{2}>0 \\
0 \text { otherwise }
\end{array}\right.
$$

Notice however that in order to demonstrate the dependence on prior information, the Bayesian analysis has also been performed for narrow and wide prior distributions for both parameters. These prior pdfs are Gamma distributions with mean equal to rough estimates of $h_{1}$ and $h_{2}$ (see Fig. 11).

Regarding the noise $\sigma$, it can be shown [25] that in order to express our complete ignorance about the size of $\sigma$, one has to choose a Jeffreys's prior on $\sigma$, i.e.:

$$
p(\sigma)=\frac{1}{\sigma}
$$

which is equivalent to saying the prior should be uniform for the logarithm of the noise (scale parameter). Finally, one can write:

$$
p(\theta, \sigma) \propto \frac{1}{\sigma}
$$

Other prior pdf's can be derived from the principle of maximum entropy. The interested reader should refer to [19, 20].

The posterior

According to Bayes's theorem (see Eq. (14)), this prior (given by Eq. (20) ) is then combined with the likelihood function to yield the posterior: 


$$
p(\theta, \sigma \mid D) \propto \sigma^{-(N+1)} \exp \left(-\frac{1}{2 \sigma^{2}} S(\theta)\right)
$$

At this point, two methods are available. Firstly, we can take into account the uncertainty about $\sigma$ by marginalizing over it. Such a marginalization is a means to cope with the systematic uncertainties. Notice that $\sigma$ is then called a nuisance parameter. In other words, $\sigma$ must be integrated out (by using the inverted gamma integral [26]) to finally yield the posterior:

$$
p(\theta \mid D) \propto S(\theta)^{-N / 2}
$$

Secondly, it is possible to consider the noise $\sigma$ as a free parameter and estimate it from the data by using a specific prior (see section 6.3).

\section{Marginal distributions}

Once the posterior pdf is computed, one can derive a (marginal) distribution for each parameter by integrating the posterior over all the other parameters (see Eq. (12)). The marginal distribution is the complete result of the Bayesian inference on the parameter of interest. Marginal posterior densities indicate the most likely values and plausible ranges for each model parameter. Obviously, a full distribution conveys much more information than a single best estimate provided by the classical approaches. Indeed, much information is lost when the inference about the parameter is summarized with one number. Firstly, the marginal distributions can be used to make straightforward comparisons between the relative plausibilities of alternative choices of parameter values. Secondly, when dealing with a highly asymmetric posterior pdf, the question of what we mean by the best estimate is rather open-ended. The maximum stills indicates the single most probable value for the parameter of interest, but the mean (or expectation) can be found to be more representative as it takes into account the skewness of the pdf. This point is reinforced when dealing with multimodal pdfs with several maxima of comparable magnitude. In this case, summarizing the inference with two or three numbers (best estimate, error-bars or even a confidence interval) cannot be done simply. In this case, the best thing to do is to just display the pdf itself [19]. This is one of the reasons for choosing the Bayesian approach to parameter estimation over maximum likelihood methods [27]

\section{Numerical implementation of the Bayesian inference}

As the number of parameters is low, the most straightforward way to evaluate the posterior pdf and its marginal counterparts is to use the simple numerical procedure that consists in evaluating the posterior for a whole series of different possible values of the parameters.

However, as mentioned in [19], it is better to compute the logarithm of the posterior and then take exponentials rather than working directly with the posterior pdf itself. As the number of measurements $N$ is rather large (a six-day hourly data experiment leads to $N=144$ ), it is preferable to work with the logarithm in order to avoid numerical problems (i.e. computer underflow or overflow). For the present application, the logarithm of the posterior of Eq. (22) is given by:

$$
L=\log _{e}[p(\theta \mid D)]=-\frac{N}{2} \log _{e}[S(\theta)]
$$

Therefore, a good procedure [19] is to evaluate the logarithm $L$ on a uniform grid of points in two dimensional $\left(X=h_{1} ; \mathrm{Y}=h_{2}\right)$ space, find the maximum $L_{\max }$ and subtract it from all the $L$ 's. Taking the exponential of these numbers will yield a value of one for the maximum of the pdf. The posterior pdf can then be displayed by using contour plots.

If normalization of this pdf is required, it can be multiplied by a constant so that the area under the curve is equal to unity. The marginal distributions are easily obtained by simply adding up the probabilities in the $X$ or $Y$ directions. For this purpose, we used the trapezoidal integration rule. As stated above, plotting the entire pdf gives us a complete picture of the inference about the value of a parameter.

\subsection{Reliabilities : best estimates and error bars}

In order to obtain a measure of the reliability of the best estimate, a Gaussian approximation of the posterior pdf is done by carrying out a Taylor series expansion of the logarithm of the posterior $L$ about the best estimate of the parameters, denoted here by the point $\theta^{*}=\left(X_{0}, Y_{0}\right)$ (see [19] for details).

This quadratic approximation yields the bivariate Gaussian for the posterior pdf:

$$
p^{*}(\theta \mid D) \propto \exp \left(\frac{1}{2} Q\right)
$$

where the quantity $Q$ is the quadratic part of the Taylor expansion that can be written in matrix notation:

$$
Q=\left(\begin{array}{ll}
X-X_{0} & Y-Y_{0}
\end{array}\right) \nabla \nabla L\left(\begin{array}{c}
X-X_{0} \\
Y-Y_{0}
\end{array}\right)
$$


with the matrix $\nabla \nabla L$ representing the Hessian of the logarithm $L$ evaluated at the point $\left(X_{0}, Y_{0}\right)$. In the present case, the second-order derivatives of $L$ were calculated by finite-differences.

Further, it can be shown [19] that the covariance matrix $C O V$ is given by the negative of the inverse of the Hessian matrix:

$$
C O V=-(\nabla \nabla L)^{-1}=\left(\begin{array}{cc}
\sigma_{X}^{2} & \sigma_{X Y}^{2} \\
\sigma_{X Y}^{2} & \sigma_{Y}^{2}
\end{array}\right)
$$

The square roots of the diagonal elements of the covariance matrix correspond to the (marginal) error-bars; the offdiagonal elements give us information about the correlations between the parameters.

\subsection{Predictive distribution of the model output: Error bars on the model output}

In the Bayesian framework, a predictive distribution of the model output can be computed. More precisely, we can assess the distribution for output $d_{k}$ by writing (and by using the preceding rules of probability):

$$
p\left(d_{k} \mid D\right)=\int_{\theta} p\left(d_{k} \mid \theta\right) p(\theta \mid D) d \theta
$$

where $p\left(d_{k} \mid \theta\right)$ is the Gaussian noise model for a datum $d_{k}$ of variance $\sigma^{2}$ and $p(\theta \mid D)$ the posterior distribution of the parameters. In order to analytically evaluate this integral, we make use of the previous Gaussian approximation of the posterior pdf (see Eq. (24)) and we approximate the model output by its linear expression around the best estimate $\theta^{*}=\left(X_{0}, Y_{0}\right)$ (see [29] for details of calculation).

Under the preceding approximations, it can be shown [29] that the integral (Eq. (27)) is easily evaluated to give a Gaussian distribution of the form:

$$
p\left(d_{k} \mid D\right)=\frac{1}{\sqrt{2 \pi} \sigma_{t}^{2}} \exp \left(-\frac{\left(d_{k}-y^{*}\right)^{2}}{2 \sigma_{t}^{2}}\right)
$$

This output distribution has a mean given by $y^{*}=f\left(\theta^{*}, t\right)$ (the model response for the optimal estimate $\theta^{*}$ ) and a variance given by:

$$
\sigma_{t}^{2}=\sigma^{2}+g^{T} C O V g
$$

One can interpret the standard deviation of the output distribution for the output as an error-bar on the mean value $y^{*}$. This error-bar has two contributions, one arising from the intrinsic noise in the data and one arising from the width of the posterior distribution.

Notice that the choice of this uncertainty propagation method was motivated by its computational advantages. In other words, this method avoids simulating the entire predictive distribution of the model output.

\section{Results and discussion}

In this section, the results of the Bayesian analysis are presented for three cases. The first one deals with the choice of a Jeffreys's prior on $\sigma$ and a flat prior for the parameters. This choice leads to the posterior pdf given by Eq. (22). In this subsection, we also show the results of the Gaussian approximation. The second case analyzes the selection of Gamma pdfs for the parameter prior distributions and the last one treats the noise $\sigma$ as an ordinary or free parameter (i.e. the noise is not integrated out).

\subsection{Jeffreys's prior on $\sigma$ and a flat prior for the parameters}

A grid with one hundred points (sufficient for the problem at hand) for each parameter allows the computation (by using the numerical procedure detailed in section 5.1) of the posterior pdf and the marginal distributions of the convective heat transfer coefficients. Notice that these computations were undertaken for hourly data over six days (i.e. $N=144$ ).

Figure 9 (a) shows the contour plot of the posterior pdf given by Eq. (22). The 2-D posterior describes our joint inference about the values of $X=h_{1}$ and $Y=h_{2}$. As seen in Fig. 9 (a), a strong correlation appears between the two estimates and the posterior pdf is slightly skewed in the upper right corner of the plot. The best estimates (maximum of the pdf) of the convective coefficients are $X_{0}=2.89 \mathrm{~W} \cdot \mathrm{m}^{-2} \cdot K^{-1}$ and $Y_{0}=0.65 \mathrm{~W} \cdot \mathrm{m}^{-2} \cdot K^{-1}$. It must also be noted that a previous work [11] based on the simplex Nelder-Mead optimization method gave the following best estimates $X_{0}=2.88 \mathrm{~W} . \mathrm{m}^{-2} . K^{-1}$ and $Y_{0}=0.60 \mathrm{~W} \cdot \mathrm{m}^{-2} \cdot K^{-1}$. 


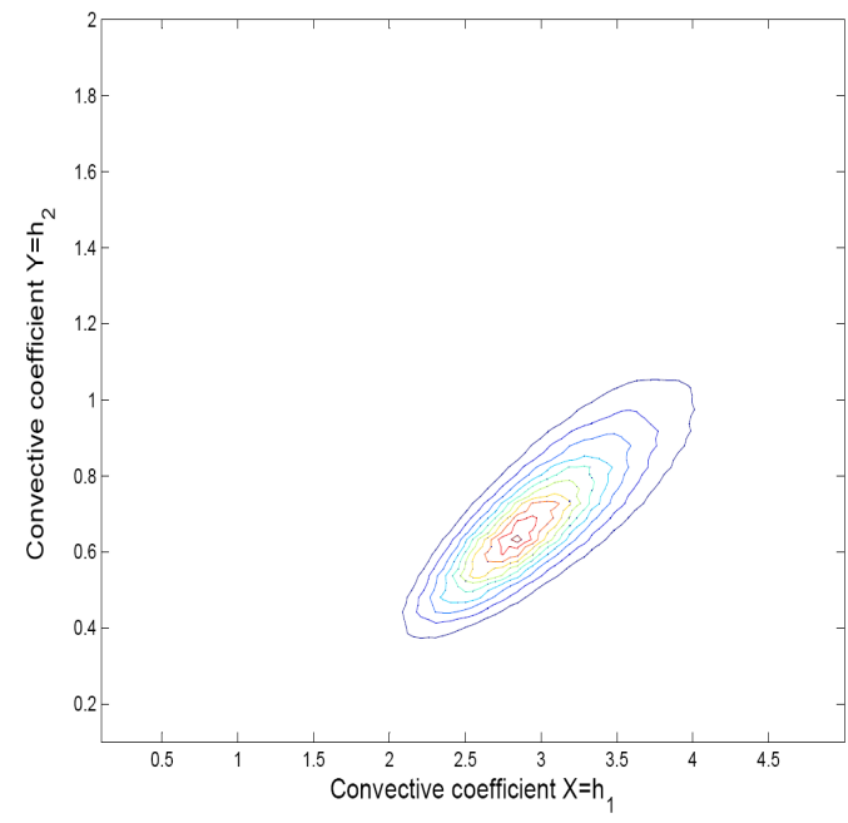

(a)

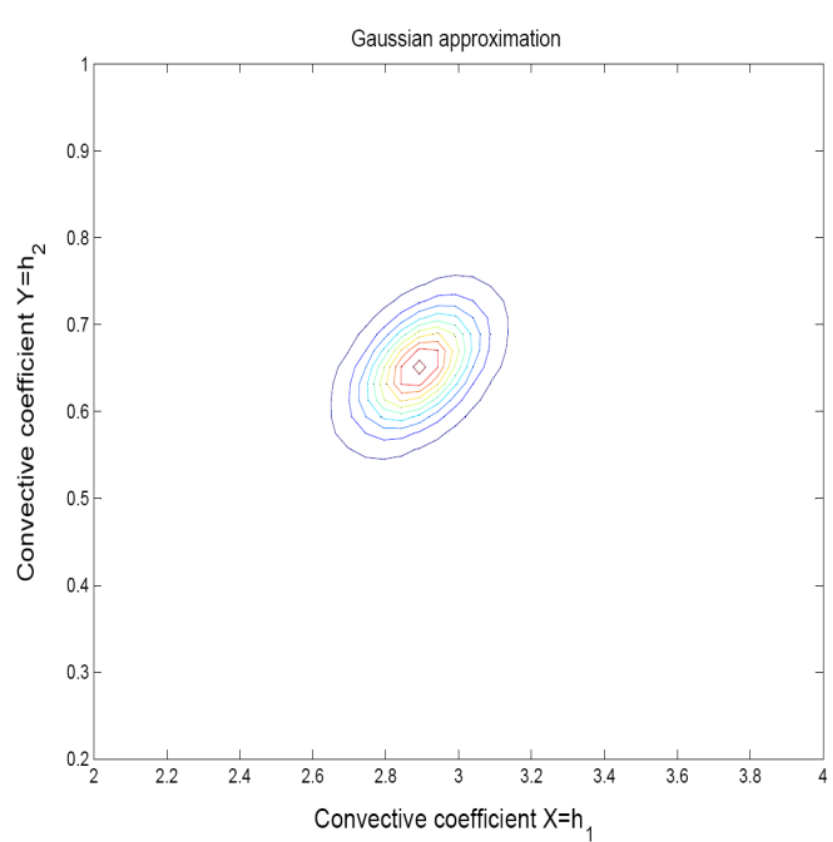

(b)

Fig. 9 Posterior pdf of $h_{1}$ and $h_{2}$ (a) and the corresponding Gaussian approximation (b). The contours correspond to $5,15, \ldots, 85$ and $95 \%$ of the maximum probability

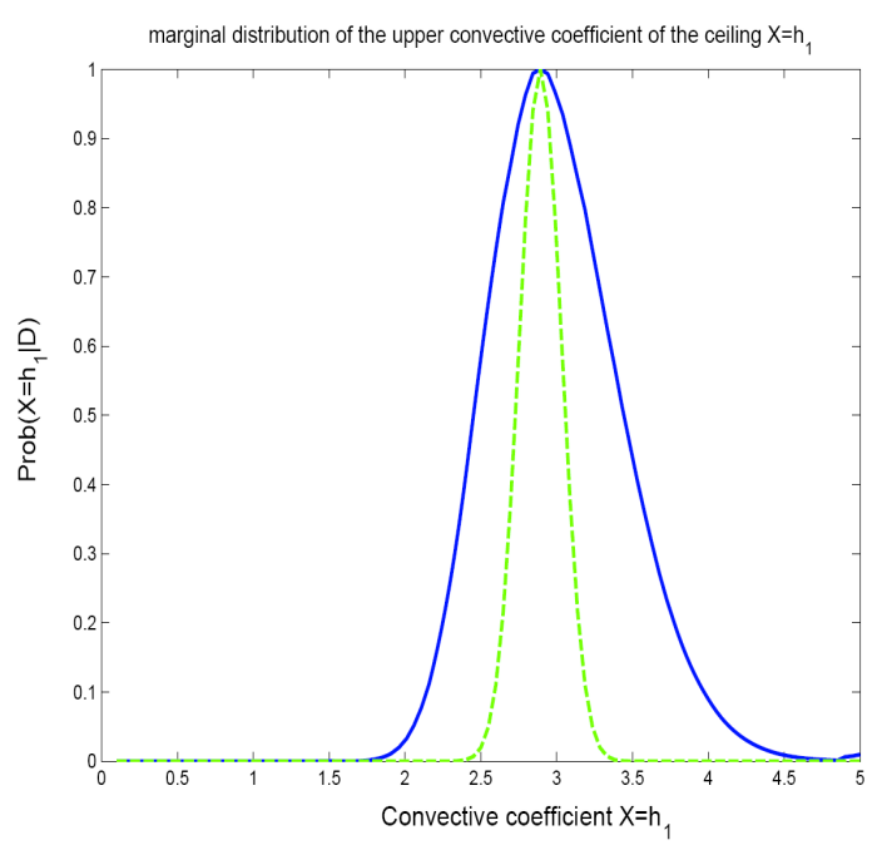

(a)

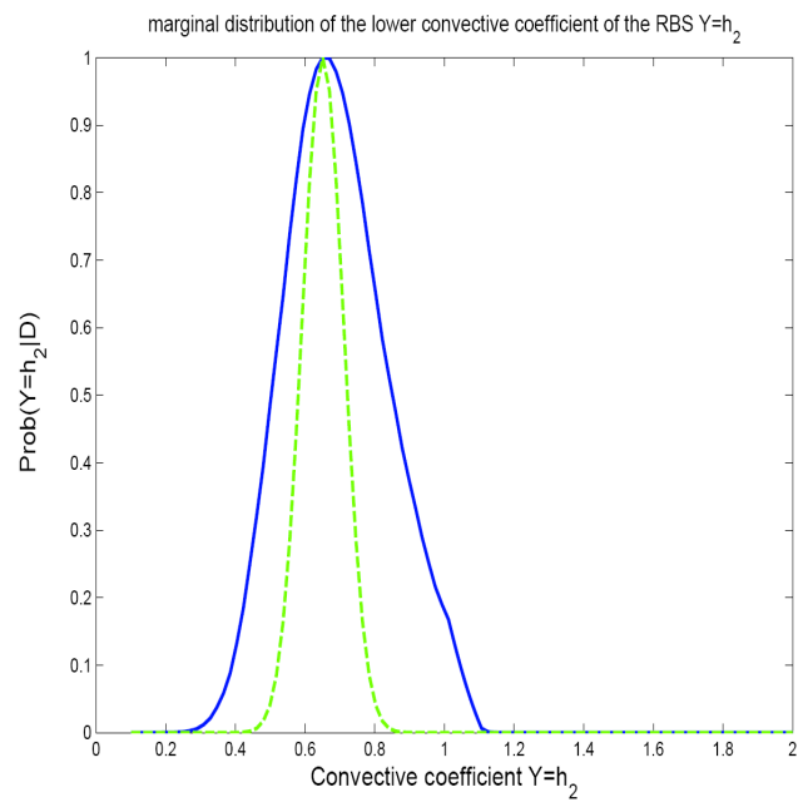

(b)

Fig. 10 Marginal posterior distributions of $h_{1}$ (a) and $h_{2}$ (b). The Gaussian approximation is shown with dashed lines.

Figure 10 represents the marginal distributions. As mentioned above, the marginal distributions have been scaled so that the greatest value of the pdf is equal to unity. As one can see, Bayesian inference gives a complete picture of the estimation problem. As illustrated by Fig. 10, a small proportion of the prior parameter space is supported by the data (for instance, in the case of $X=h_{1}$, values below 1.5 or above 4.5 are very unlikely). In particular, the base case values of $h_{c i}=1 \mathrm{~W} . \mathrm{m}^{-2} . K^{-1}$ (see lines 5 and 6 of Table 2) inferred from the correlation given by Eq. (2) have a very low probability. The greater 
uncertainty on $X=h_{1}$ points towards the difficulty in estimating this parameter and confirms the important role played by this coefficient (see results of the sensitivity analysis). Here, its contribution to the output uncertainty is greater than the other coefficient.

\section{Gaussian approximation}

Figure 9(b) shows the Gaussian approximation of the posterior distribution (see Eq (24)). Notice that the range of the parameters has been restricted to between $2<\mathrm{X}<4$ and $0.2<\mathrm{Y}<1$. The corresponding marginal distributions are plotted in Fig. 10 as dashed lines. As expected, the quality of the Gaussian approximation is good in the neighborhood of the best estimate. However, one may notice that this approximation underestimates the uncertainties associated with the parameters. Hence, although it is sometimes desirable (and necessary) to summarize the inference about the values of the parameters with just a few numbers (best estimates, correlations and error-bars), it is clear that some information is lost in the process.

Computation of the covariance matrix according to Eq. (26) leads to the following respective error-bars for each parameter: $\sigma_{X}=0.14 \mathrm{~W} \cdot \mathrm{m}^{-2} \cdot K^{-1}$ and $\sigma_{Y}=0.06 \mathrm{~W} \cdot \mathrm{m}^{-2} \cdot K^{-1}$.

The value of the covariance term is $\sigma_{X Y}^{2}=+0.0038$, which shows that the two inferred parameters are correlated (the correlation ratio $r=\frac{\left|\sigma_{X Y}^{2}\right|}{\sigma_{X} \sigma_{Y}}$ has a value of 0.46). Further, as $\sigma_{X Y}^{2}>0$, there is a correlation between the inferred parameters, which means an over-estimate of one is accompanied by an over-estimation of the other. This fact may render the analysis somewhat disappointing (i.e. we would prefer de-correlated parameters) but these are the conclusions that can be derived from the available data. As stated by [19], the real cure is to improve the characteristics of the posterior pdf by collecting more relevant data, or by supplementing it with cogent prior information (i.e. a more informative prior instead of the flat prior used in the analysis).

\subsection{Gamma prior distributions for the parameters}

In this subsection, we address the question of choosing different prior pdfs for the parameters (see Fig. 11). The Bayesian analysis was therefore repeated with two alternative prior pdfs. The first alternative consists in a narrow Gamma distribution for both parameters. A narrow prior pdf expresses good prior knowledge of the parameters. The second alternative proposes a wide prior Gamma distribution which conversely expresses poor prior knowledge of the parameters. Table 4 lists the parameters (mean and variance) of the Gamma distributions.

Table 4 Parameters of the prior Gamma distributions

\begin{tabular}{cc}
\hline \hline Prior & Gamma distribution $\Gamma(s, c)$ \\
\hline $\begin{array}{c}\text { Narrow prior for } \\
h_{1}\end{array}$ & Mean sc ; variance $s^{2} c=0.01$ \\
Narrow prior for & Mean $s c=1$; variance $s^{2} c=0.001$ \\
$h_{2}$ & \\
Wide prior for & Mean $s c=2.5$; variance $s^{2} c=2$ \\
$h_{1}$ & \\
Wide prior for & Mean $s c=1$; variance $s^{2} c=0.2$ \\
$h_{2}$ & \\
\hline \hline
\end{tabular}




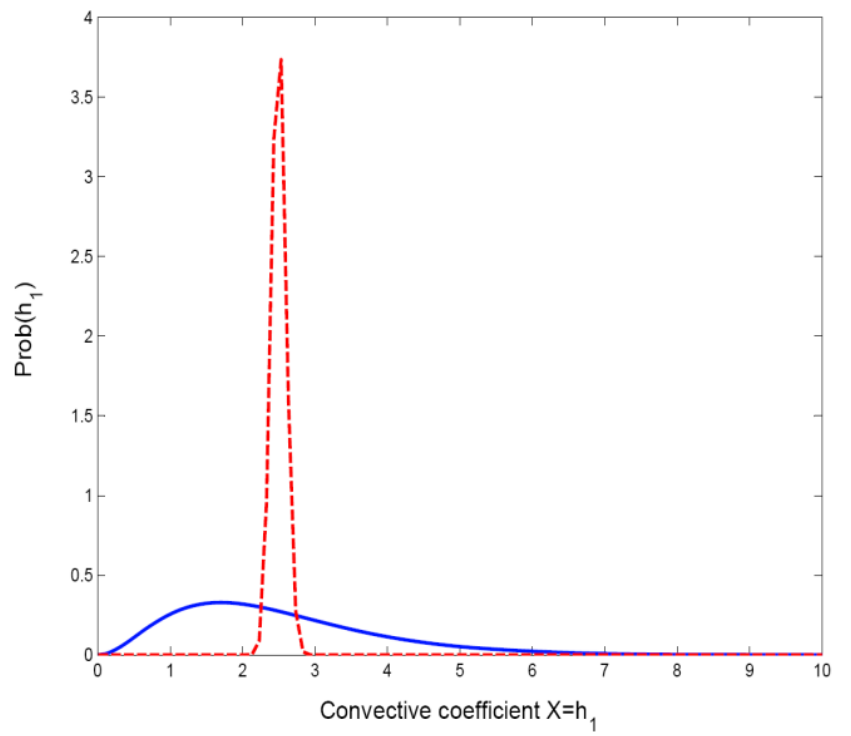

(a)

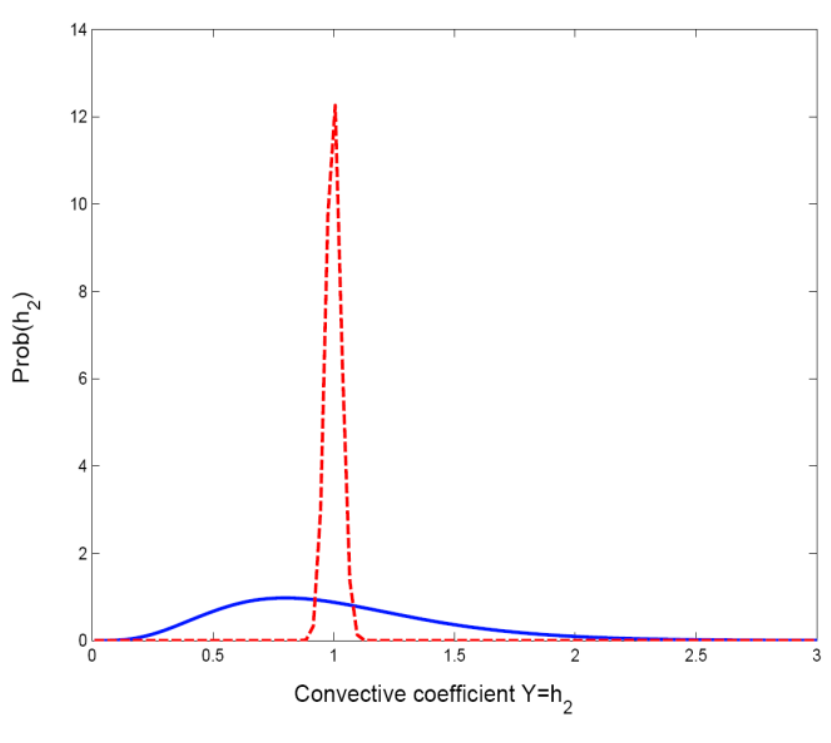

(b)

Fig. 11 Gamma prior distributions for $h_{1}$ (a) and $h_{2}$ (b). The wide prior is shown with a solid line.

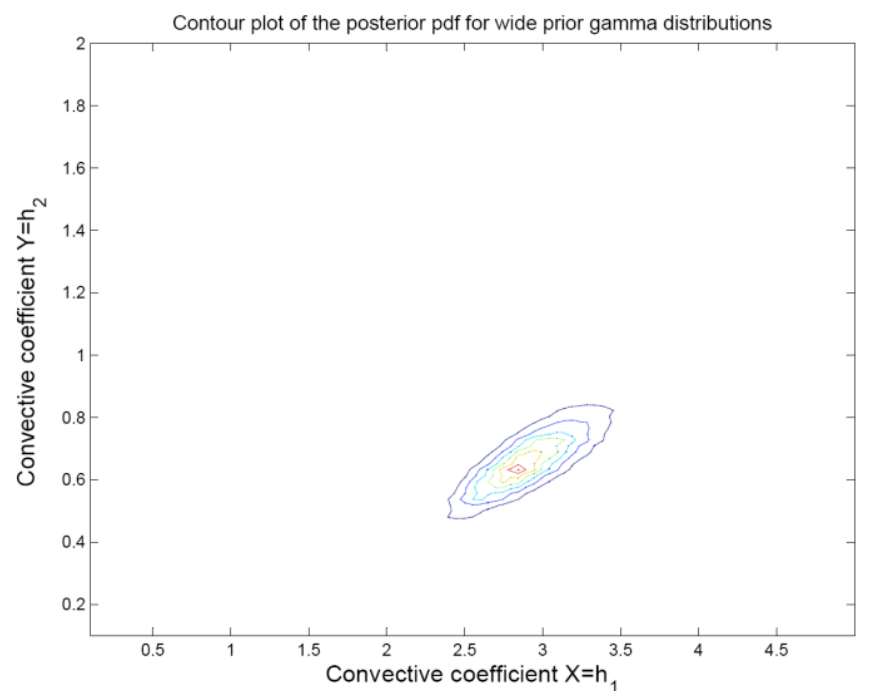

(a)

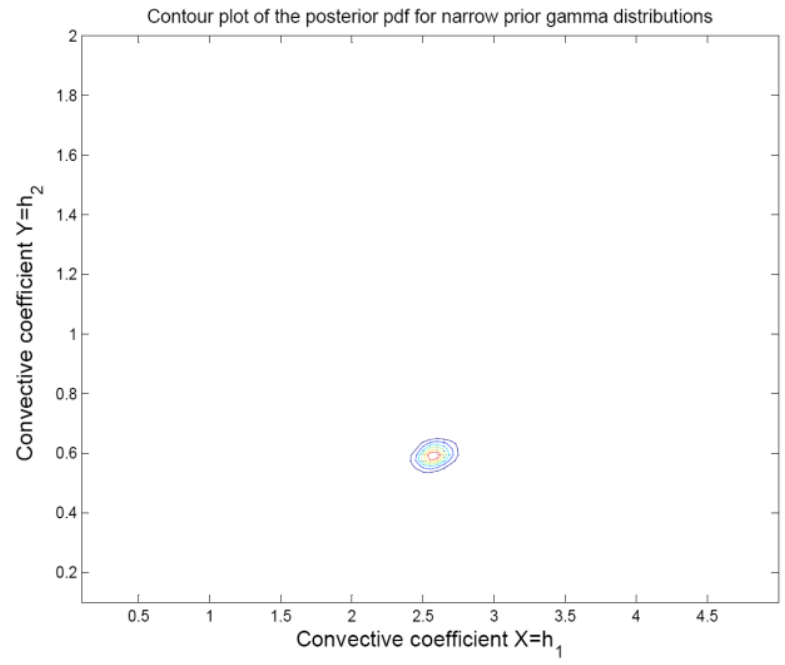

(b)

Fig. 12 Posterior pdf of $h_{1}$ and $h_{2}$ for the case of wide Gamma prior (a) and narrow Gamma prior (b) 


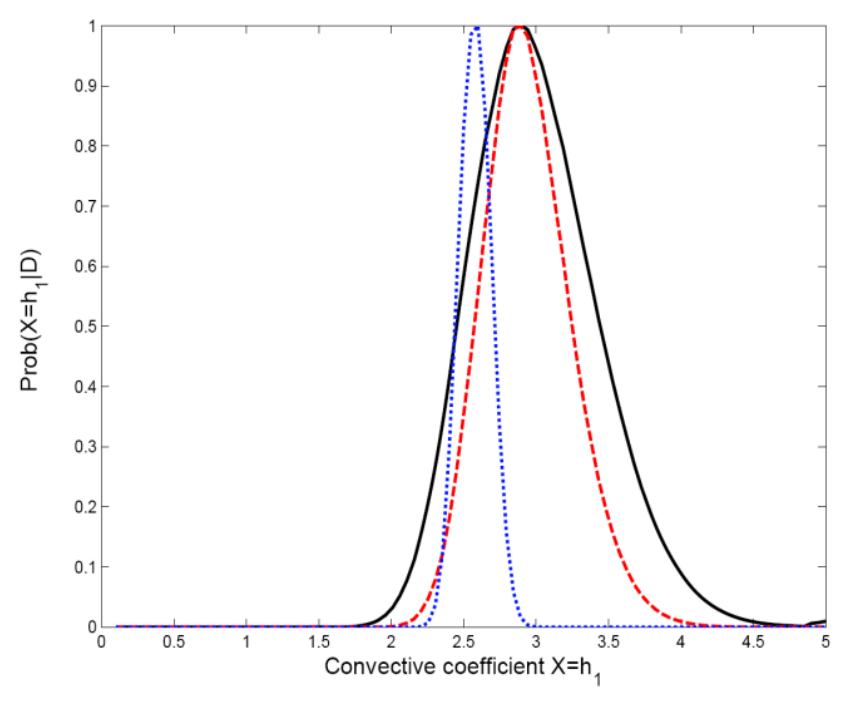

(a)

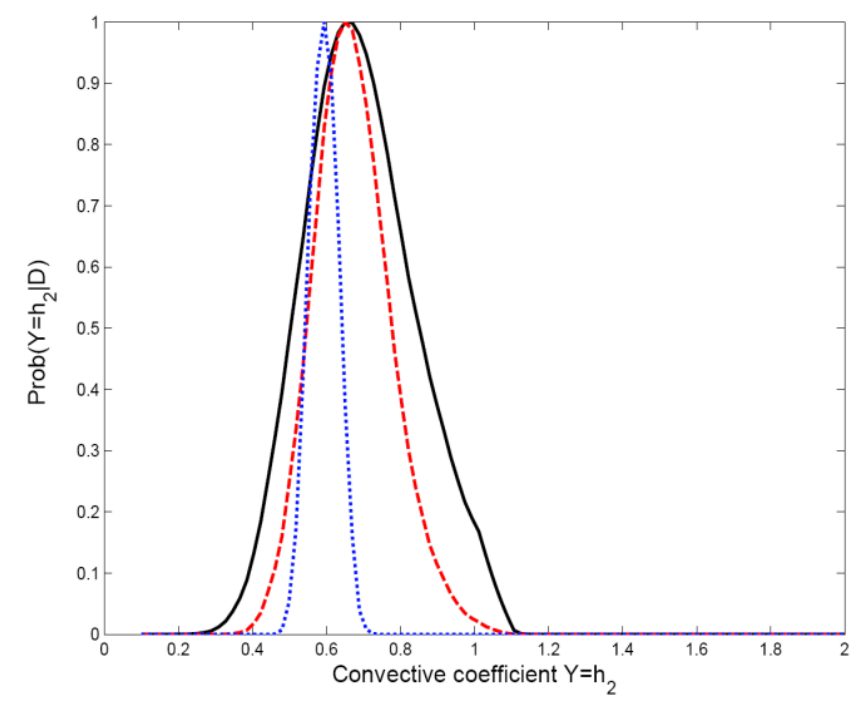

(b)

Fig. 13 Marginal posterior distributions of $h_{1}$ (a) and $h_{2}$ (b). The solid lines correspond to a flat prior for the parameters; the dashed lines correspond to the wide prior Gamma distributions and the dotted lines correspond to the narrow prior Gamma distributions.

\section{Good prior knowledge of the parameters}

In this situation, the best estimates are $X_{0}=2.59 \mathrm{~W} \cdot \mathrm{m}^{-2} \cdot K^{-1}$ and $Y_{0}=0.59 \mathrm{~W} \cdot \mathrm{m}^{-2} \cdot K^{-1}$. In the case of $X=h_{1}$, the prior information dominates the likelihood function. If more data could be collected, the Bayesian estimates would converge with each other and with the maximum likelihood estimate. Put differently, as the empirical evidence grows, the analysis would lead to the same conclusions (i.e. to the same best estimates), irrespective of our initial beliefs. Conversely, regarding the parameter $Y=h_{2}$, one can conclude that the evidence of the data is strong. Indeed, the maximum of the pdf seems to evolve (rapidly) towards $Y_{0}=0.65 \mathrm{~W} \cdot \mathrm{m}^{-2} \cdot K^{-1}$. Regarding the uncertainties, as seen in Fig. 12(b), the uncertainty of the parameters in the posterior distribution is determined by the prior pdfs. Moreover, the correlation between parameters is very small because of the dominating influence of the independent prior distributions (we recall that independence was assumed between parameters, see Eq. (17)). As a conclusion, one can expect to obtain good (de-correlated) estimates with small posterior uncertainty by using cogent prior information and by collecting relevant data on the process.

\section{Poor prior knowledge of the parameters}

As expected, the selection of the wide Gamma prior distributions yields a wider posterior distribution and a greater correlation between the parameters. The posterior is then dominated by the likelihood function and the best estimates are the same as those inferred from a flat prior for the parameters i.e. $X_{0}=2.89 \mathrm{~W} \cdot \mathrm{m}^{-2} \cdot K^{-1}$ and $Y_{0}=0.65 \mathrm{~W} \cdot \mathrm{m}^{-2} . K^{-1}$. One may notice however that the inferences (see the corresponding marginal pdfs in Fig. 13 (dashed lines)) are better that those obtained with a flat prior. This fact tends to promote the use of an (even poor) informative prior over the use of the classical flat prior.

\subsection{The noise $\sigma$ is a free parameter}

As mentioned above in section 5.1, instead of integrating out $\sigma$, it is possible to consider the noise $\sigma$ as a free parameter. In this case, the noise $\sigma$ is estimated from the data by using a Gaussian prior of mean 0.5 (which corresponds to the classical thermocouple measurement error assumption of $0.5^{\circ} \mathrm{C}$ ) and standard deviation of 0.05 . Notice that Gamma or lognormal pdfs are typically chosen as distributions for positive parameters such as, in this case, the standard deviation, but in the present case the use of a Gaussian prior does not create any real problems as the prior has very negligible probability on negatives values.

The marginal distribution of $\sigma$ (fig. 14) indicates that the noise is well-determined from the data with a plausible range of approximately 0.7 to 0.9 and a best estimate of 0.81 . This latter result tends to confirm that the noise comprises measurement noise plus modeling error. Furthermore, the contour plots (not shown) of $\left(h_{1}, \sigma\right)$ or $\left(h_{2}, \sigma\right)$ exhibit no correlation between the noise $\sigma$ and the parameters $h_{1}$ and $h_{2}$. 


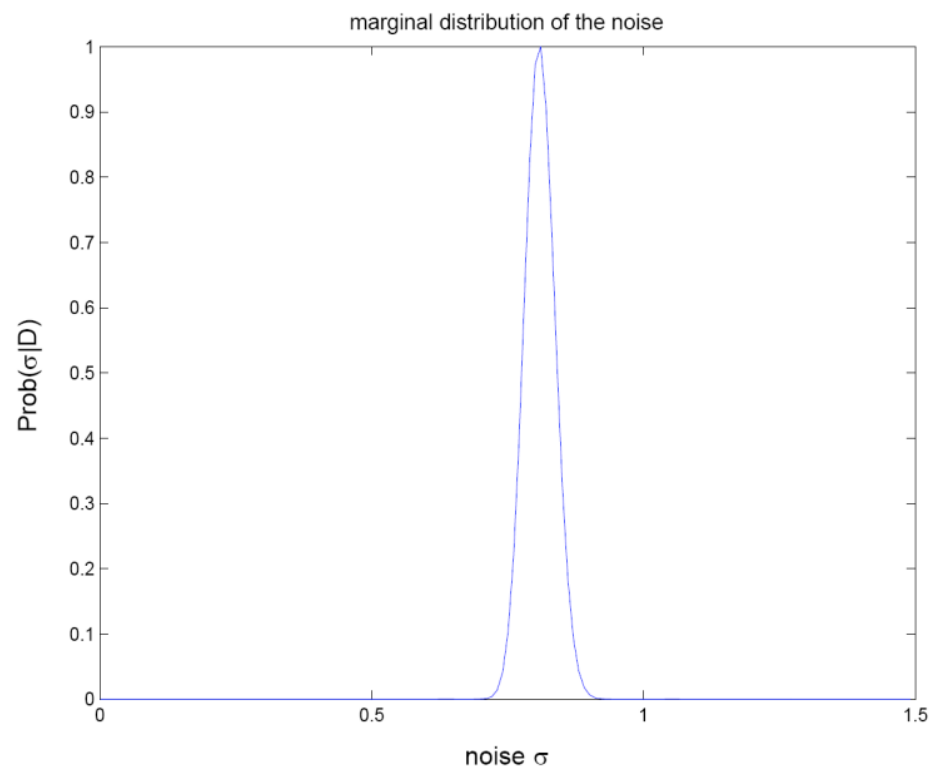

Fig. 14 Marginal posterior distribution of the noise $\sigma$.

\subsection{New appraisal of the model}

The appraisal of the model with the values given by the best estimates $X_{0}=2.89 \mathrm{~W} \cdot \mathrm{m}^{-2} \cdot K^{-1}$ and $Y_{0}=0.65 \mathrm{~W} \cdot \mathrm{m}^{-2} \cdot K^{-1}$ is illustrated in Fig. 15 .
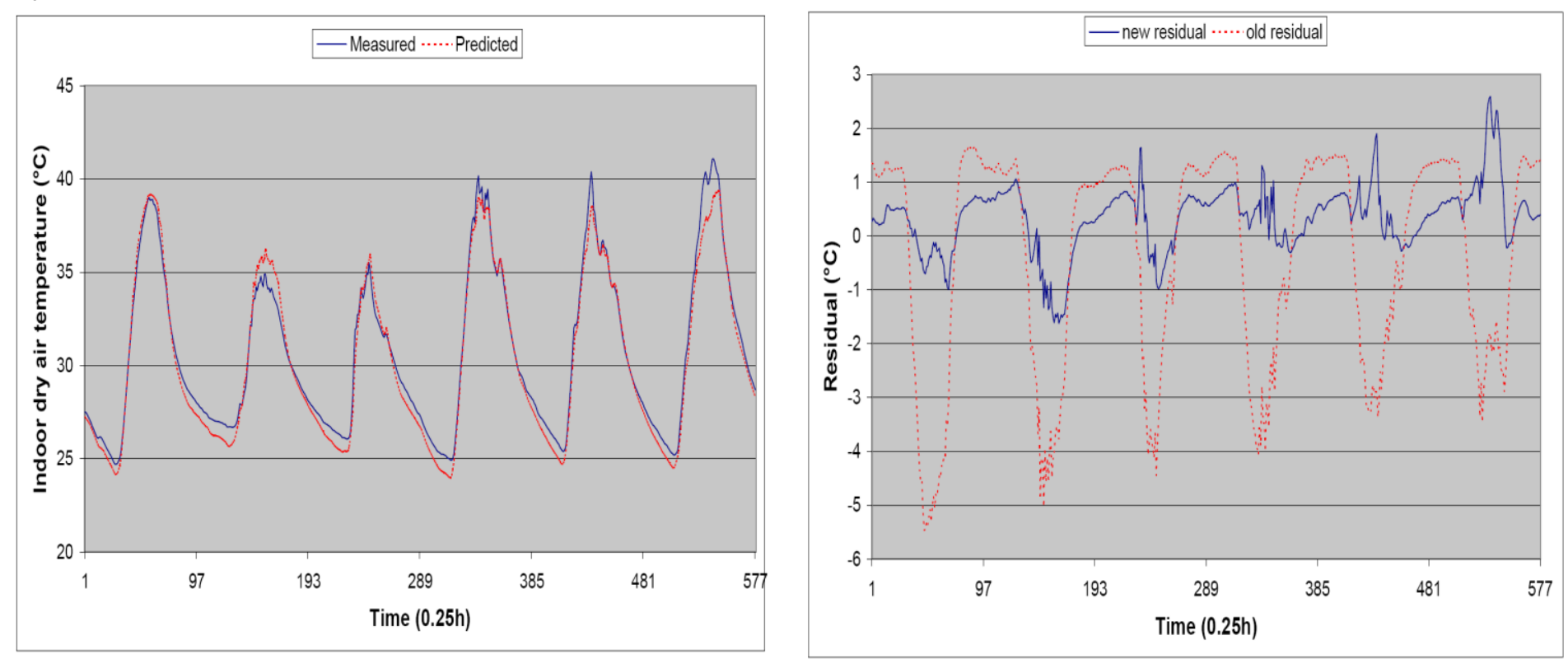

Fig. 15 Comparison between measurement and prediction of the indoor dry air temperature of the lower air layer $T_{l o}$. The old residual corresponds to the one depicted in Fig. 6.

New values for the convective heat transfer coefficients have clearly improved the model. The mean value of the residuals of $T_{l o}$ is $0.34{ }^{\circ} \mathrm{C}$ and its standard deviation is $0.62{ }^{\circ} \mathrm{C}$. Conversely, no significant improvement is observed for $T_{a i}$. We recall that the old residuals correspond to the appraisal of the model depicted in section 3.5 (see Fig. 6).

It can be noted that the model's predictive ability has been improved as a result of being calibrated to the data, but that different standards should be applied for judging the quality of fit of a model depending on whether the model has been calibrated with the data or not. 


\subsection{Checking model validity}

The Bayesian approach is interesting as it leads naturally to the derivation of a model prediction uncertainty interval. As discussed above, computation of model output uncertainty intervals is necessary for checking model validity.

We recall that the tool proposed by Task 22 of IEA relies on Monte Carlo simulations which can be computationally demanding in order to infer this uncertainty interval. Further, in this process, a priori uncertainty intervals are assigned to the parameters. For instance, the model uncertainty intervals may be given by the variation of $10 \%$ about the nominal value of the parameters.

Conversely, the Bayesian approach offers a flexible and natural framework and sound theoretical basis for assessing uncertainties in model parameters and uncertainties in model output. All subsequent analyses follow directly from the posterior parameter distributions. In our opinion, the use of actual posterior information provided by the Bayesian approach may constitute a good alternative to characterize model uncertainty intervals.

As mentioned in section 5.3, these parameter uncertainties together with the noise in the measurements lead (see Eq. (29)) to an error-bar on the output. In order to compute this error-bar, the value of the noise has been replaced by its best estimate of 0.81 inferred from the data.

Figure 16 shows a significant overlapping between uncertainty measurement and simulation intervals and therefore tends to confirm the validity of the new model. Note that the measurement uncertainty interval has been set to $\left[-1^{\circ} \mathrm{C}+1^{\circ} \mathrm{C}\right]$.

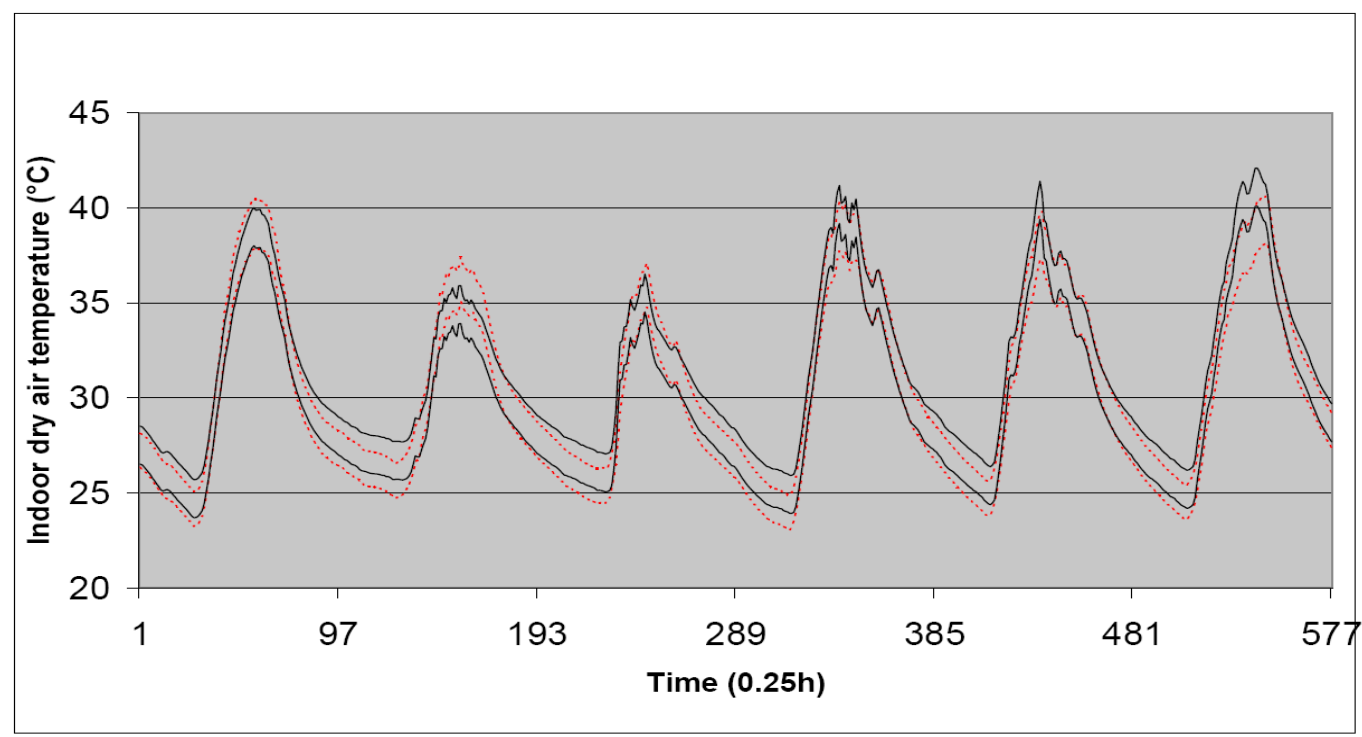

Fig. 16 Comparison between measurement uncertainty intervals (continuous line) and prediction intervals (dotted line)

In the case where error bars on the output are too large compared to the measurement uncertainty bands, one can say that the model is less helpful. One can also say that more high quality data are needed in order to correctly infer the parameters and their associated uncertainties.

Finally, such a method offers a diagnosis of the building thermal model. Indeed, following the idea given in [6], large discrepancies observed between nominal and estimated parameters confirm the need to properly design or review the heat convection model in the lower air layer.

\section{Conclusion and perspectives}

An empirical validation exercise on a thermal model of a roofing complex highlighted the need to carefully tune two convective heat transfer coefficients of the lower air layer of a roofing complex that included a radiant barrier.

This two-parameter example, though simple, gives a concrete illustration of the Bayesian inference approach to the estimation of convective heat transfer coefficients.

Application of Bayes's theorem allows the derivation of the posterior pdf of the parameters and the associated marginal pdf's. The latter indicate the most likely values and plausible ranges for each model parameter. The best estimates (maximum of the marginal pdf) of the inferred parameters greatly improve the building thermal model of the roofing complex.

Reliabilities for each parameter have been assessed and a corresponding error-bar on the model output has been derived. Thus, this methodology helps as it leads naturally to the derivation of a model prediction uncertainty interval. Further, the comparison between the model prediction interval and the measurement uncertainty interval tends to confirm the validity of the new model. 
The sensitivity of the Bayesian analysis has also been assessed for different prior distributions. It appears that the use of even rough prior information can noticeably improve the characteristics of the posterior pdf.

As a conclusion, we can say that Bayesian methods provide explicit means of incorporating uncertainty into modeling and forecasting and a corresponding measure of reliability for the conclusions reached.

It seems that the results could be improved by obtaining more relevant data, or by making the analysis with cogent prior information. The principle of maximum entropy (MaxEnt) $[19,20]$ may help to assign this prior probability.

\section{Acknowledgment}

The authors are grateful to the anonymous reviewers for their helpful comments.

\section{Nomenclature}

$A \quad=$ square matrix

$B \quad=$ matrix

$C=$ diagonal matrix

$C O V=$ covariance matrix

$D=$ set of experimental measurements

$d \quad=$ experiment measurement

$f \quad=$ driving frequency assigned to a parameter

$g \quad=$ gradient of the model output

$H \quad=$ matrix

$h=$ convective heat transfer coefficient $\left(\mathrm{W} \cdot \mathrm{m}^{-2} \cdot \mathrm{K}^{-1}\right)$

$k=$ Simulation number

$L=$ logarithm of the posterior

$m \quad=$ Number of simulation time steps

$n \quad=$ Number of simulation runs

$N \quad=$ number of experimental measurements

$p \quad=$ probability density function (pdf)

Prob = probability or probability density function

$Q \quad=$ quadratic part of the Taylor expansion of the logarithm

$r=$ correlation ratio

$S=$ sum-squared of the residuals

$t=$ time

$T \quad=$ temperature $\left({ }^{\circ} \mathrm{C}\right)$

$X=$ first parameter

$Y \quad=$ second parameter

$y=$ model response

$z \quad=\quad$ standardized parameter for the sensitivity analysis

$Z \quad$ = parameter for the sensitivity analysis

\section{Greek}

$\Delta T=$ temperature difference at the surface-air interface $\left({ }^{\circ} \mathrm{C}\right)$

$\nabla \nabla L=$ Hessian matrix of the logarithm of the posterior

$\beta_{i}=$ first-order regression coefficient

$\beta_{i j}=$ second-order regression coefficient

$\lambda=$ oscillation amplitude

$\varepsilon=$ noise, emissivity or error term

$\theta=$ vector of parameters

$\sigma \quad=$ standard deviation of the noise $\left({ }^{\circ} \mathrm{C}\right)$

$\Gamma \quad=$ Gamma distribution

\section{Subscripts}

$0=$ related to the optimal estimate or the nominal value

$1=$ upper convective coefficient of the ceiling

$2=$ lower convective coefficient of the RBS

ai $=$ indoor dry air temperature

base $=$ base case value

ci $=$ indoor convective coefficient

$\mathrm{i}=$ related to the $\mathrm{i}^{\text {th }}$ parameter 
$\mathrm{j} \quad=$ related to the $\mathrm{j}^{\text {th }}$ parameter

$\mathrm{k} \quad=$ related to time $t_{k}$

lo $=$ lower air layer of the roofing complex

$\mathrm{t}=$ related to the standard deviation on the output

up $=$ upper air layer of the roofing complex

\section{Superscripts}

* $\quad=$ optimal or Gaussian posterior pdf

$0=$ nominal value

\section{References}

[1] Medina, M. A., 2000, “On the performance of radiant barriers in combination with different attic insulation levels," Energy and Buildings, 33(1), pp. 31-40.

[2] Al-Asmar, H. R., Jones, B., W., and Matteson, D., 1996, "Experimental Evaluation of Attic Radiant Barriers (RP-577)," ASHRAE transactions, 102(1), pp. $297-306$.

[3] Fairey, P., 1985, "The measured side-by-side performance of attic radiant barrier systems in hot and humid climates," Proc. 9th International Thermal Conductivity Conference, Cookville, Tenn., pp. 481-496.

[4] Hall, J. A. , 1985, "Performance testing of radiant barriers," Proc. 3rd Annual Symposium on Improving Building Energy efficiency in hot and humid climates, Arlington Texas, pp 55-77.

[5] Palomo del Barrio, E. and Guyon, G. , 2003, "Parameters space analysis tools for empirical model validation. Part I.

Theory and computer implementation," Energy and Buildings, 35 (10), pp. 985-996.

[6] Palomo del Barrio, E. and Guyon, G. , 2002, "Using parameters space analysis techniques for diagnosis purposes in the framework of empirical model validation," Final Report, Task 22: Building Energy Analysis Tools, International Energy Agency.

[7] Bretthorst, G. L., 1990, “An introduction to parameter estimation using Bayesian Probability," Maximum Entropy and Bayesian Methods, P. Fougere, eds., Kluwer Academic Publishers, Dordrecht the Netherlands, pp. 53-79.

[8] Miranville, F., 2002, "Mise en place d'une plate-forme expérimentale - réalisation et instrumentation d'un dispositif de caractérisation d'isolants minces réfléchissants, " $\mathrm{PhD}$ thesis, University of La Réunion., France.

[9] Boyer, H., Garde, F., Gatina, J.C., and Brau, J. , 1998, “A multimodel approach to building thermal simulation for design and research purposes," Energy and Buildings, 28 , pp. 71-78.

[10] Boyer, et al., 1996, "Thermal building simulation and computer generation of nodal models," Building and environment, 31 , pp. 207-214.

[11] Miranville, F., Boyer, H., Mara, T., and Garde, F. , 2003, "On the thermal behavior of roof-mounted radiant barriers under tropical and humid climatic conditions: modelling and empirical validation," Energy and buildings, 35, pp. 997-1008.

[12] Cole, R.J., and Sturrock, N. S., 1977, "The convective heat exchange at external surface buildings," Building and environnment, 12, pp. 207-214.

[13] Fauconnier, R., and Grelat, A., 1981, "Thermique de l'habitat : bases de la modélisation thermique ," Annales de l'I.T.B.T.P, 395, pp. 123-138.

[14] Mara, T. A., Boyer, H., and Garde, F., 2002, “ Parametric Sensitivity Analysis of a Test Cell Thermal Model Using Spectral Analysis," Transactions of the ASME, Journal of Solar Energy Engineering, 124, pp. 237-242.

[15] Malakoff, D.M., 1999, "Bayes offers 'new' way to make sense of numbers," Science 286, pp. 1460-1464

[16] Cox, R. T., 1946, "Probability, frequency and reasonable expectation,” Am. J. Phys., 14, pp. 1-13.

[17] Bayes, T., 1763, “An essay towards solving a problem in the doctrine of chances," Phil. Trans. Roy. Soc., 53, pp. 370418.

[18] Laplace, P.S. de, 1812, Théorie analytique des probabilités, Courcier Imprimeur, Paris.

[19] Sivia, D.S., 1996, Data analysis: a Bayesian tutorial, Oxford University Press, Oxford, UK.

[20] Jaynes, E.T., 2003, Probability theory- The logic of science, Cambridge University Press, Cambridge, UK.

[21] Jaynes, E.T., 1986, "Bayesian methods: an introductory tutorial," Maximum entropy and Bayesian methods in applied statistics, Cambridge University Press, Cambridge, UK.

[22] Loredo, T.J. 1990, "From Laplace to supernova 19871: Bayesian inference in astrophysics," Maximum entropy and Bayesian methods in applied statistics, Cambridge University Press, Cambridge, UK.

[23] Bretthorst, G.L., 1990, "Bayesian model selection: Examples relevant to NMR," Maximum Entropy and Bayesian Methods, P. Skilling, eds., Kluwer Academic Publishers, Dordrecht the Netherlands, pp. 377-388.

[24] Qian, S.S., Stow,C.A. and Borsuk, M.E., 2003, “On Monte Carlo methods for Bayesian inference,” Ecological modelling, 159, pp. 269-277.

[25] Jeffreys, H. 1939, Theory of probability, Clarendon Press, Oxford, UK.

[26] Seber, G.A.F and Wild C.J., 1989, Non linear regression, Wiley and sons, New York. 
[27] Ellison, A.M., 1996, "An introduction to Bayesian inference for ecological research and environmental decision-making," Ecological applications, 6(4), pp. 1036-1046.

[28] D’Agostini, G., 2003, "Bayesian inference in processing experimental data - Principles and basic applications," Reports on progess in Physics, http://www.roma1.infn.it/ dagos

[29] Bishop, C.M., 1995, Neural networks for pattern recognition, Oxford University Press, Oxford, pp. 398-401, Chap. 10. 